\title{
Della quantità nel vocalismo milanese
}

1.0. Se la quantità vocalica, dopo il crollo delle sue opposizioni nel latino, si è ridotta nel campo romanzo a mero fenomeno combinatorio, e fonologicamente rilevante essa non è oggi che nel friulano e in determinati dialetti italiani settentrionali (Tekavčić 1972: 22 nota), il milanese è da annoverarsi fra tali dialetti? Ossia, ha valore pertinente la quantità vocalica che caratterizza il vocalismo milanese odierno? Quali sono, poi, le evoluzioni fonetiche che ne possono aver determinato la comparsa? Questi alcuni fra i quesiti principali che il presente lavoro si pone, e che cerca di chiarire alla luce delle descrizioni del vocalismo milanese tuttora disponibili.

1.1. Una delle caratteristiche più cospicue del sistema vocalico latino era la quantità dei singoli fonemi vocalici, ereditata dall'indoeuropeo, fonologicamente rilevante, e indipendente dalla struttura sillabica. Associata alla quantità come tratto concomitante era, per un periodo più o meno lungo, una differenza nel grado d'apertura, per cui le vocali lunghe venivano pronunciate chiuse, e aperte le brevi (ad eccezione di Ǎ) (Straka 1959: 286-9; Väänänen 1963: 29; Wartburg 1967: 48-9; Sidney Allen 1965: 47). A questo sistema che utilizzava distintivamente le opposizioni quantitative (PŌPǓLUS 'pioppo' contro PǑPǓLUS 'popolo,' ŌS ‘bocca' contro ǑS 'osso,' RŌSĂ [nom.] contro RŌSĀ [abl.], ecc.) subentrarono progressivamente, in modo difforme $\mathrm{e}$ in momenti diversi a seconda delle regioni, sistemi basati sulle opposizioni qualitative che stanno alla base dei vocalismi delle lingue romanze attuali.

1.2. Da alcune delle ipotesi finora avanzate per render conto di tale passaggio sembrerebbe che la quantità vocalica latina, una volta defonologizzata, fosse del tutto scomparsa da tutti i sistemi: ciò che viene sostenuto, ad es., da Haudricourt e Juilland che pure concordano con il Wartburg (1965 : 48-9) nell'ammettere uno stadio intermediario preromanzo, caratterizzato dalla presenza contemporanea della quantità e del timbro, susseguente a quello latino a base di quantità. Secondo questi studiosi $\left(1946_{2}: 31-42,45\right)$ la quantità vocalica sarebbe venuta, per un periodo, ad essere assente, anche foneticamente, dal sistema romanzo occidentale, per ricomparirvi verso il VI secolo come preludio alla diversificazione delle toniche a seconda della struttura sillabica (tonica lunga in sillaba libera, breve in sillaba implicata).

1.3. Contro gli autori dell'Essai, afferma il Weinrich $\left(1969_{2}: 181\right)$ che "Den Quantitätenkollaps als Verlust der Vokalquantitäten aufzufassen, wäre ein Irrtum." Sostiene, infatti, il Weinrich che la quantità latina sia stata defonologizzata in seguito alla soppressione prima della sequenza voc. lunga + 
cons. lunga (a causa di una Unzuräglichkeit nel sistema quantitativo latino (Weinrich $1969_{2}$ : 18), poi di quella voc. breve + cons. breve mediante l'allungamento o l'abbreviamento delle rispettive toniche o consonanti, cosi da conformarsi alle rimanenti due sequenze di voc. lunga + cons. breve e voc. breve + cons. lunga. La quantità vocalica, che prima era indipendente dalla struttura della sillaba, in quanto la vocale lunga poteva trovarsi sia in sillaba libera che in quella implicata, viene, in seguito a questa trasformazione, ad essere prevedibile dalla struttura sillabica, la tonica lunga potendosi ora avere solo in sillaba libera, e la breve in quella implicata. Poiché allungandosi in sillaba libera e abbreviandosi in quella implicata le toniche mantengono il proprio grado d'apertura originario, una nuova opposizione, basata, appunto, sul grado d'apertura viene a sostituirsi a quella basata sulla quantità vocalica (Weinrich $\left.1969_{2}: 36\right)$.

Secondo il Weinrich $\left(1969_{2}\right.$ : 183), la quantità originaria latina, non più fonologicamente rilevante, rimane presente come tratto ridondante anche dopo il crollo delle quantità latine, e continua fino ad oggi (ma solo foneticamente, si badi) in quegli idiomi romanzi nei quali non si può parlare di una diversificazione della tonica a seconda della struttura sillabica (TÉLA, toscano tẹla). ${ }^{2}$

1.4. La quantità che caratterizza il vocalismo milanese odierno non è da collegarsi direttamente con la quantità latina, che del resto non è stata conservata da nessun idioma romanzo. ${ }^{3}$ Essa è, dunque, secondaria, posteriore, e di formazione relativamente recente.

2.0. Fra le descrizioni più o meno dettagliate del vocalismo milanese verranno qui di seguito discusse, nell'ordine di successione, quelle del Biffi (Lepschy 1978; si veda la nota 48), del Cherubini (1839), del Rajna (1881), del Salvioni (1884, 1911, 1919, 1975), di Isella (1959), di Beretta (1980), e di Nicoli (1983). Omesse sono quelle di Pagani (1945), di Angiolini (1897) perché troppo sommarie, e quella di Pavia (1928) che non si è avuto modo di consultare.

2.1.1. Il Biffi distingue le vocali larghe (largh) da quelle strette (strec): una distinzione, questa, che pare sia basata su una differenziazione quantitativa per tutte le vocali, sia quantitativa che qualitativa per alcune di esse ( $a$, $e, o[u])$. Le vocali larghe sono brevi, le strette sono lunghe; trattandosi, però, di $a, e, o[u]$, sono brevi e aperte le prime, lunghe e chiuse le seconde. ${ }^{4}$ Alle vocali larghe susseguono le consonanti aspre o gagliarde (asper, ghaiard), a quelle strette le consonanti dolci o vuote (dolz, loìi). La distinzione fra consonanti aspre o gagliarde e dolci o vuote corrisponde con ogni probabilità a quella fra consonanti forti (per lo più sorde), scritte doppie, ${ }^{5}$ e deboli o leni (per lo più sonore).

2.1.2. Il sistema vocalico del Prissian comprende i seguenti fonemi vocalici: [å:] pas PACE, nas, corà COLLĀRE, prà, ghauasg, masg MAIU, par 
PALU, i participi passati in $-a<-\bar{A} T U$, ecc.; [a] ma MAGIS, la, à HABE(T), strauasc <*EXTRAVACUARE, pann, gli infiniti in -à <-ĀRE, ecc.; [e:] pes

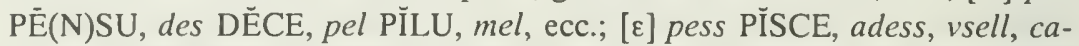
pell, pern PINNNA, ecc.; [u:] (grafia $o)^{6}$ spos; [u] loss TŬSSE; [0] porch; [i:] dis $\operatorname{DICl}(\mathrm{T})$; [i] diss $\mathrm{DIXI}(\mathrm{T}) ;[\mathrm{y}:]$ vegnù, vedù ${ }^{7}[\emptyset:]$ choùr, fioù, voùna. ${ }^{89}$

La serie delle nasali comprende (escluse [ø], [๑] che non sono mai nasalizzate) le toniche 'strec,' cioè lunghe per [ī:], [ỹ:], lunghe e chiuse per [ã:], [ẽ:], [ü:]: vùn, pan, fen FÉNU, bon BŎNU, son SŎNU, SUM, ecc.

2.2.1. Il Cherubini (1839: XXVII-XXIX, XXXIV-XXXV oppure 2243$45,2247-8$ ) distingue tre varianti ("suoni" nella sua terminologia) per i fonemi vocalici $a, i, u[\mathrm{y}]$ : vibrato, rimesso, stemperato, i cui valori è difficile determinare con precisione, ma che corrispondono con ogni probabilità ai gradi di lunghezza, ${ }^{10}$ indicate rispettivamente con l'accento grave, l'assenza di segni, e il raddoppio, e illustrate dai seguenti esempi: stà 'stare,' pà 'padre,' mì 'io o me,' sì 'sì,' dì 'di,' virtù; sta 'questa,' ciama 'chiama,' mi 'a me,' comunal; andaa, trii, veduu.

Le tre varianti ("suoni" del Cherubini) si riducono a due per oeu[ø], vibratissima la prima (indicata con accento circonflesso), e rimessa la seconda (senza alcun segno, o con accento grave), per le quali cita toêे 'togli,' 'prendi,' e bonoeur, i toeù 'i tuoi.'

2.2.2. Per $e$, il Cherubini distingue due gradi d'apertura $(\dot{e}, \grave{e})$ con correlazioni di quantità non chiaramente riconoscibili. ${ }^{11}$ Se -ée sta per [e:] in sillaba finale libera, come in asée, stée che il Cherubini riporta, però, come esempi di é chiusa, non evidente risulta il valore quantitativo di é in réd, més, ecc. che trovo nel Vocabolario. Che, però, si tratti di una [e:] anche in questi ultimi esempi, si può arguire dalla correlazione che, come si vedrà più avanti, esiste nel milanese fra il grado d'apertura e la quantità (cfr., ad es., § 2.4.16.). Esempi di [ $[\varepsilon]$ sono mè, tasè (e sètt, mètt nel Vocabolario). Ma se [ $\varepsilon:]$, viene notata con una dieresi in sillaba chiusa (oh dëss 'ohibò'), forse con -èe in sillaba finale libera (bebèe, accanto a bè, 'agnello').

Maggiori difficoltà presenta il caso di $o$, chiusa in dolór, onór (con “. . . due altri suoni particolari ... l'uno vibrato, e per lo più ne' monosillabi come in sô (sole), ${ }^{12}$ l'altro stemperato come in soo (io so). Accenno il primo coll'accento circonflesso; il secondo colla doppia vocale." [Cherubini 1839: XXVIII o 2243]); aperta in còr 'coro,' òr 'oro.'

2.2.3. Non affatto chiaro risulta da tutto ciò come sia distribuita la quantità delle vocali, secondo che queste si trovino in parossitonia o ossitonia, in sillaba libera o (divenuta) implicata, o se esista una correlazione fra il grado d'apertura e quello di durata. Chiaramente riconoscibile non è la quantità vocalica se non in sillaba libera finale che il Cherubini rappresenta, appunto, $\mathrm{col}$ raddoppiare la vocale interessata (tranne oeu), diacriticamente segnandola, per i timbri $e$ ed $o$, per specificarne la qualità chiusa (asée ACETU, coó 
CAPU(T)) od aperta (limitatamente ad $e$ in pochissimi casi: bebè (accanto a $b \dot{c}$ ) 'agnello'),' ${ }^{13}$ altrimenti scrivendo sempre -àı, -ii, -ìu (andàa, sentii, vediu). Per le altre posizioni sussistono incertezze, per le quali non ci soccorrono l'astrusità e l'inadeguatezza dei termini vibrato, rimesso, stemperato c l'uso incoerente che ne fa il Cherubini.

2.2.4. Se si accoglie l'interpretazione che di questi termini dà il Rajna (cfr. nota 10), facendo valere l'equipollenza vibralo-breve (perché decisamente brevi sono le vocali in sı̀̀, pà, ecc., esempi che il Cherubini cita, appunto, per i suoni vibrati), e supponendo che il Cherubini abbia fatto uso degli stessi segni diacritici (cui si è fatto riferimento sopra) anche in sede alfabetica, si verrebbe ad avere, all'interno di parola, sotto l'accento, solo ed esclusivamente $a, i, u[y]$ vibrate, cioè brevi, perché segnate con accento grave in pàs, màr, fil, amis, dìr, vocali tutte che il Salvioni invece considera lunghe, trascrivendo pās, mär, fill, amig, dür (Salvioni 1884: passim). Quanto ai timbri $e$ ed $o[\mathrm{u}]$, l'accento acuto o grave in réd RÉTE, més ME(N)SE, pél PĬLU, gésa EC(C)LËSIA, vérd VĬR(Ī)DE, crós CRŬCE, nós NŬCE, nevód NEPŌTE, pèss PISSCE, pèll PĔLLE, còrp CǑRPU, ecc. (Cherubini 1839: s.v.) ne indicano, a quanto pare, solo il grado d'apertura, senza fornire alcuna informazione sulla loro relativa durata, ma, poiché il Cherubini differenzia per essi il grado chiuso da quello stemperato (cioè lungo), e limitatamente ad $o$ il grado chiuso da quello vibrato (se vibrato vale breve: cfr. nota 12), è lecito supporre che é ed ó negli esempi citati qui sopra rappresentino vocali

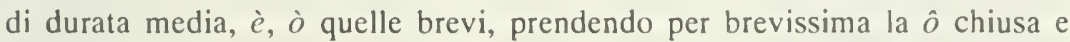
vibrata in sô, ecc. (cfr. nota 12), e per atona la rimessa (cfr. nota 11).

2.2.5. Di fronte a tali dubbi, non è possibile farsi un 'idea esatta sulla durata delle vocali milanesi in base alla descrizione del Cherubini. Tutt'al più, si può dire che, mentre esse possono essere lunghe o brevi in sillaba finale libera (andàa, andà, sentii, senti, cùu, vertì), non ben determinato resta il loro valore all'interno di sillaba, quindi di parola.

2.2.6. Parlando ancora dei suoni prolungati che il milanese possiede a dovizia "nelle sillabe finali delle voci," il Cherubini (1839: XXIX o 2245) nota: "Altri di tali suoni per fine sono simili a quelli che sentonsi nelle voci italiane pronunziate aperte, ma con questo che dove l'italiano batte il suono su la vocale, il milanese lo stempera e ne strascica picciola parte su la successiva consonante. Così Ràna, Sèna, Mìna, Suòna, Lìna sono da noi proferite con quello stemperamento di suono che sentesi nei nostri plurali Rann, Minn, Lıın, ecc.; e di qui è invalso l'uso generale di segnare questo suono col raddoppiare anche nel numero del meno le consonanti susseguenti a sì fatte vocali. Scrivonsi Pàppa, Grècca, Scimma, Euròppa le voci Papa, Greca, Cima, Europa, e Campanna, Estrèmma, Cadènna, Berlinna, Corònna, Vìnna le voci Campana, Estrema, Catena, Berlina, Corona, Una, le quali s'hanno poi a pronunziare non già sdoppiando le consonanti come farcbbe l'italia- 
no, ma facendo sonar la vocale antecedente insieme con esse a quel modo ch'ei farebbe se avesse a pronunziarne i plurali Campann, Estremm, Cadenn, Berlinn, Coronn scritti in questa guisa. Inutilità, se vuolsi, perché non è la consonante che si raddoppii, ma sì la vocale che, pronunziata apertissima, dà un tal suono; pure è inutilità di uso invalso e si lascia correre." Come sempre, non poche le difficoltà d'interpretazione in cui ci si imbatte davanti al passo citato, ma alla luce di quanto asserisce altrove lo stesso Cherubini (1839: 2246) a proposito della $n,{ }^{14}$ sembra si possa dedurne che egli riconoscesse un certo rafforzamento per alcune consonanti (le sorde e le nasali, come appare dagli esempi riportati), soprattutto quando finali. Pare, inoltre, di poterne dedurre che la pronuncia forte della consonante, dalla posizione finale, dove era originariamente sorta, si fosse a suo tempo e per via analogica estesa anche alla posizione intervocalica, susseguente a sillaba penultima.

2.3.1. I suoni distinti dal Cherubini in vibrato, rimesso, e stemperato per il Rajna corrispondono alle tre categorie di vocali brevi, medie e lunghe, esemplificate da fà, ciallad, veritaa; pè, spêd, pee, goss, occôr, poo; finna, rid, vorii; brutt, rûd, luu; foeura, foeugh, fioeu (plur.) che poi vengono ridotte in due, ". . . comprendendo nella categoria delle lunghe anche le medie, che in sostanza le appartengono..." (Rajna 1881: 11). Una tale corrispondenza, in realtà se c’è, è difficile vedere (cfr. § 2.2.4).

2.3.2. Gli esempi riportati dal Rajna sembrano suggerire che di media durata sono suscettibili d'essere le vocali in sillaba tonica finale uscente in consonante semplice (o in certi gruppi di consonanti), ma che, ai fini di una bipartizione quantitativa, si possono includere nella categoria delle lunghe che, non esclusivamente, possono stare in sillaba libera tonica finale. II Rajna stesso ci dà la seguente norma: “. . . sono brevi le vocali seguite da una doppia o da certi gruppi di consonanti, lunghe quelle seguite da una consonante semplice o da certi altri gruppi. Si noti, per esempio, la lunga di sporg, incorg, confort. Quanto alle vocali in fin di parola, parte sono brevi parte lunghe, a seconda dell'origine." (Rajna 1881:11).

2.3.3. Per quanto concerne $e, o$, il Rajna ci dice che $e(\overline{\mathrm{E}}, \breve{\mathrm{I}})$ è aperta quando seguita da consonante "più o meno doppia," da gn, da gruppi di consonanti di cui la prima sia s (scenna, menna, ingegn, colmegna, medemm, insemma, mansuett, mett, giughett, pess, istessa, bellezza, fregg, oreggia, todesch, cresta), nelle terminazioni degli infiniti della coniugazione II (avè, vedè, piasè); chiusa (e nasalizzata) davanti a $-n$ finale (leggermente nasalizzata davanti a $n$ complicata) (ben, presenza, dent, vend, scendera, ecc.), davanti a $m$ complicata (temp, november); che $o(\overline{\mathrm{O}}, \overline{\mathrm{U}})$ è aperta davanti a $m$, mm, gn, "t (personna, besogn, vergogna, nomm, Romma, rott, sott, nagotta (eccezioni insomma, bott 'botte'); aperta ancora, perché discendente da Ŏ, in pocch, socca, foss, or, confort, sporg, ma chiusa in mocch 'mozzo,' mocc 'mozzicone di sigaro,' bocca, ross, occor, descors (Rajna 1881: 10-11). 
2.3.4. Si ha una correlazione fra la durata e il grado d'apertura: “ l'a lungo volta la sua faccia dalla parte dell'o sulla bocca di chi parla sbottasciaa; tanto più, quanto maggiore la lunghezza." (Rajna 1881: 11).

2.4.1. E' difficile riassumere in poche righe la descrizione di gran lunga più particolereggiata che del sistema vocalico milanese ci ha lasciato il Salvioni nei suoi vari contributi (Salvioni 1884, 1911, 1919, 1975). In Fonetica (Salvioni 1884), egli distingue le vocali $a, i, \ddot{o},{ }^{15} \ddot{i}, c,{ }^{16}$ e tre gradi di $o$ e di $\mathcal{c}$ : $o$ (simbolo $o),{ }^{17} e$ chiuse, $\dot{o}, \grave{e}$ aperte e $o, e$ che sarebbero intermedie fra le aperte e le chiuse, per cui non dà esempi, ma che potrebbero corrispondere alle atone delle stesse. Per le vocali toniche $a, i, o, e$, ed $\ddot{i}$ si ha la nasalizzazione per effetto della $-n$ finale.

2.4.2. Dalle descrizioni del vocalismo milanese, e dagli sporadici riferimenti che vi fa l'eminente studioso in varie sedi, sj deduce che sono passibili d'essere lunghe

i) le vocali toniche in sillaba finale libera;

ii) le vocali toniche in sillaba finale uscente in consonante originariamente o attualmente sonora.

2.4.3. Per quanto riguarda la posizione (i), il Salvioni (1911: 386) nota: "... il milanese lascia cadere il $-r$ e il $-l$ dopo vocale accentata e . . questa risulta allora lunga. ..." Risultano ancora lunghe le desinenze del participio passato che si riflettono oggi per -aa, -ıu, -ii, <-ATU, -AT1, -ATAE, ecc. (cantaa, l'eduu, sentii). ${ }^{18}$ Lunghe pure le toniche, divenute finali, dei sostantivi participiali (straa, contraa), dei sostantivi quali fraa, fiaa, praa; quelle che risultano dalla contrazione dei dittonghi secondari (cantee, vedii, sentii CANTATIS, CANTATE, ecc.; da -ATE (-AS, -ATIS) reritaa, ecc.

2.4.4. Non ostanti tutti questi casi ed altri ancora che vi si possono aggiungere, riesce impossibile formulare norme precise circa l'esito, lungo o breve, delle toniche finali secondarie, avendosi nel dialetto, accanto a veduu, sentii, ecc., veriu, mari, padü PALŪDE, pedii PEDŪLE (Salvioni 1884: § 188) che invece presentano, con le loro $-\grave{u}$, -i brevi, uno sviluppo nel senso inverso.

2.4.5. A ciò si aggiunga che " . . ha una genesi e una vicenda diversa il $-r$ dell'infinito debole (il forte perde l'intiera sillaba ‘ere già in Bonvesin ... ), il quale, cadendo, non lascia lunga la precedente tonica (mil. cantá, vedé, senti)." (Salvioni 1911: 386). Quali fattori abbiano, poi, portato all'abbreviamento nelle uscite degli infiniti quando altrimenti risultano in prevalenza lunghe le toniche precedenti la $-r$ finale romanza, è difficile determinare. Il Salvioni (1911: 386-7) crede che le finali degli infiniti fossero brevi già al tempo di Bonvesin. A questa supposizione egli giunge dalle rime bonvesiniane descenderà / montar / guardar / remirar (C 9-12), recuintar / ha / darà / peccar (O 169-72), ia / retornar / dexdegniar / far (L 117-20), ecc. (cui si 
aggiunga mi / fi / menti / obedhi (T 655-8): cfr. Contini 1941: XXIX) alle cui forme rimanti con la desinenza -ar (cioè-à) dell'infinito il milanese moderno fa corrispondere una - $a$ breve, e dalla contrapposizione di queste rime a quelle perfette (tipo $-a(e)(<-\mathrm{ATE}) /-a(l)$ ), quale utilitae / caritae / amistae / celestial (B 281-4). Lo stesso Salvioni osserva, però, altrove che sono lunghe le finali degli infiniti quando seguite dai pronomi enclitici $-g,-f: \operatorname{lod} \bar{a} f$, rubāg, vedêf 'vedervi,' vedēg 'vederci,' vêf, vēg 'avervi, avergli,' sentif, dāg, dìg, dîf, ecc.; brevi, invece, quando seguite da altri pronomi: vedèll, vedèj, sentimm, lodànn, setass, ecc., notando la differenza fra i primi (comincianti con consonanti sonore o originariamente sonore) e i restanti pronomi in -ll, $-m m,-n n$, ecc., e affermando alla fine: "Il tutto si compendia nella legge che, in generale la vocale d'uscita sparendo dopo consonante sonora rese lunga la vocale tonica della sillaba precedente." (Salvioni 1884: 278). Dal ragionamento del Salvioni si è indotti a credere che fossero originariamente brevi le finali degli infiniti per poi allungarsi all'appoggiarvisi di enclitici in consonante sonora. Si può con altrettanto fondamento sostenere l'ipotesi che esse fossero originariamente lunghe (per cui lodā $f$, lodāg) e che siano successivamente riuscite a brevi dai frequentissimi casi di enclisi dei pronomi in $-n,-m,-l$, ecc. che a loro volta si saranno poi rafforzate, come di solito avviene quando sono precedute da tonica breve. A conferma di ciò, gioverà qui osservare che anche la desinenza $-\vec{e},-\bar{\imath}$ dell'imperat. $2^{\mathrm{a}}$ pers. plur., da lunga normalmente (mandē, senti) o quando seguita da - $g$, -f (mandēg 'mandategli,' dervig 'apritegli,' salvêf 'salvatevi') diviene breve in mandemm 'mandatemi,' sentill 'sentitelo,' ecc.

2.4.6. Per la posizione (ii) (ved. § 2.4.2.) il Salvioni (1884: 159) ci dà la seguente norma: “ . . . in milanese, la vocale d'uscita, sparendo dopo consonante sonora, rese lunga la vocale accentuata di penultima." (cfr. § 2.4.5). ${ }^{19}$

Va subito notato che l'ortografia tradizionale milanese continua a rappresentare la sonora, anche se questa non rispecchia più la pronuncia reale, sia per ragioni etimologiche (in quanto, a mo' d'esempio, roeud [rö:t] 'ruote' ha accanto la forma del singolare roeuda, l'accrescitivo rodon; ved [ve:t] le altre forme del paradigma, che presentano la sonora vedi 'vedo,'veden 'vedono,' vedeva, veduu, vedè, ecc.), sia per il rapporto che intercorre tra la vocale lunga e la sonora (originaria) susseguente, da cui “ . . . risultò che pel milanese lo scrivere la media fosse quasi un segno grafico per esporre la quantità della vocale che la precedeva: röt è pel milanese röt (con ö breve) ${ }^{20}$ "rutto" ma röd è röd, set è sét "sei tu o sai tu» sed è séd «sete»" (Salvioni 1884: 159). ${ }^{21}$

Altri casi in cui la sonora finale (solo grafica!) indichi la quantità della tonica precedente si hanno (Salvioni 1884: passim) in $f \overline{o ̈ g}$, lög, gö̈g, crēed, nevōd, ecc., e ancora (Cherubini 1839: s.v.) moeuv 'muovere' nev 'neve,' rav 'rape,' ecc. che il Salvioni (1884: 160) scrive, eccezionalmente, mö $f, n \bar{e} f$, rāf, ecc., nonostante la tonica lunga. ${ }^{22}$ 
Si badi, tuttavia, che non tutte le consonanti sonore che vengono a trovarsi in uscita si convertono alle sorde corrispondenti, come mostrano forme quali pas PACE, pes PE(N)SU, maz MAIU, pe $2 \mathrm{P} E \mathrm{IU}(\mathrm{S})$ (Salvioni 1884: $\$$ 162, 166) che con la loro sonora contrastano con quanto afferma il Salvioni.

2.4.7. D’altra parte, generalmente brevi ed aperte, anzi apertissime, sono le toniche di parole attualmente o originariamente parossitone, in sillaba susseguita da consonante attualmente (e originariamente) sorda, nasale, tavolta liquida, raramente sonora (solo grafica, che in realtà della pronuncia si desonorizza). Interviene in questi casi la "posizione milanese," termine che il Salvioni (1884: 156) adotta per riferirsi a una particolarità, assai controversa, della fonetica milanese.

2.4.8. Se le vocali, nelle condizioni suddette, divengono aperte e brevi, le consonanti, a loro volta, che l'ortografia tradizionale milanese suole rappresentare con la doppia, vengono pronunciate in modo che (e qui il Salvioni (1884: 156-7) cita il Rajna (1881: 9)) “ . . . l'alfabeto italiano non ci permette di ben rappresentare né con una $n$ sola (il Rajna parla qui più precisamente di $n$ ma il ragionamento può valere per tutte le consonanti che si trovano nelle condizioni di cui sopra) né con due, sebbene in mancanza di meglio, si sia pur costretti ad adottare l'uno o l'altro partito. Il femminile di bon (bō) non è né bona, né bonna letti all'italiana. L'n di questi casi è vibrata come la doppia toscana, ma più breve e compatta; ché, invece di ripartire le sue articolazioni tra la vocale antecedente e la seguente, le appoggia per intero alla seguente, quasi fosse scritto bo-rma. E nella stessa posizione suonano analogamente per ragioni analoghe, anche altre consonanti; insemma, gne-cca, e-cco (eco), Euro-ppa, poe-tla."

2.4.9. Che il Salvioni riconoscesse pienamente la rilevanza fonetica di questo fenomeno per il vocalismo milanese, non ostanti le riserve che esprime riguardo al termine da lui stesso adottato per designarlo (" . . . Ia posizione milanese termine improprio all'uopo comeché piuttosto che d'un fatto reale di posizione trattisi d'una geminazione puramente ortografica invalsa nell'uso ad esprimere il fenomeno che qui ci occupa." [1884: 156]) è sufficientemente provato da quanto afferma altrove (1884: 153): "la posizione milanese intacca sempre più o meno la qualità della vocale cui essa segue: $\grave{e}$ per $i$ ed $e$, ò per $o, e$ o un suono molto affine per $\ddot{i}$," oltreché dagli appositi paragrafi che ad esso fenomeno consacra nella trattazione dei singoli fonemi vocalici. ${ }^{23}$

2.4.10. Come si rileva dalla definizione stessa, le liquide $-l,-r$ (soprattutto questa ultima) non vanno sempre soggette al rafforzamento consonantico (perché di questo pare che si tratti), ciò che è comprovato da forme quali fill, pél, (Salvioni 1884: §§21, 33) azāl (accanto a azā) *AC1ALE, inguāl (accanto a inguā), ecc. (Salvioni 1884: § 188), rūr, cūir, ciūr, fiōr, dü̈r, mü̈r, ecc. (Salvioni 1884: passim). Fra quelle forme in cui il fenomeno, invece, si manifesta, il Salvioni (1884: passim) cita müll MŪLU, parolla PARA(B)ǓLA, 
tolla TA(B)ŬLA, soll SŌLU, vell VĒLU, cortell CŬLTELLU, pell, quell, stella, e carr, ferr, corr, ecc. Ma in queste ultime forme (carr, ferr, corr) la doppia è solo grafica, come poi nota il Salvioni (1884: 161: “ . . che in realtà suonano cār, fer (è lungo) cōr . .. "; anche tōr TŬRRE (Salvioni 1884: $\S 55) .{ }^{24}$ Tenuto conto di ciò, e del fatto ancora che la $r$ complicata non fa sempre posizione (Isella 1959: 649) e che la tonica che la precede, a quanto pare, è lunga (cfr. § 2.3.2.) (cfr. anche Salvioni 1919: 525, n.1), è molto probabile che la grafia $r r$ sia soltanto etimologica, e che, quindi, non rappresenti alcun rafforzamento della liquida. Per quanto concerne la liquida $l$, d'altra parte, pare che si debba partire dalle forme in cui, in seguito allo sviluppo neolatino, essa è pervenuta a trovarsi in posizione finale. È noto che, in questa posizione, la $l$ è spesso soggetta a cadere. Questa sua labilità, aggregata al fatto che sovente si rotacizza (gora GULLA, mere, tore *TOLLI(T), ecc. in Bonvesin) (e si palatalizza davanti ad una - $i$ finale, fondendosi con essa; ciò che avviene anche in posizione interna) testimonia di una sua articolazione debole o lene. La tonica precedente questa $-l$, a causa stessa del suo carattere lene, deve essersi presto allungata. ${ }^{25}$ Che essa fosse lunga già al tempo di Bonvesin, il Salvioni è incline a credere, prova sufficiente essendone le rime -ae (<-ATE) / -al, - co / -el, ecc. nei volgari bonvesiniani, che corrispondono alle $-a a,-c e$ lunghe del milanese odierno, e la contrapposizione di queste a quelle cui il milanese odierno fa invece corrispondere la $-a$ breve (Salvioni 1911: 386-7) (cfr. § 2.4.5.).

2.4.11. Pare, tuttavia, che anticamente, parallela a questa, ci corresse un'altra tendenza, forse sorta sotto l'influsso analogico della $-l(l)$ - discendente dalla -LL- geminata, e rivolta invece a conservare la -l finale romanza. Questa $-l$, in seguito ulteriormente rafforzatasi, avrà agito sulla tonica precedente per abbreviarla. Così, infatti, ci si potrà rendere conto del diverso esito, nello stesso contesto fonetico, in cuu CŪLU con [y:] lunga (-l ammutolita) da un lato, e in mull MŪLU con [y] breve (-ll rafforzata) (analogamente soll SŌLU) dall'altro.

2.4.12. Per le forme coll, moll, cavall, vall, quell, scigolla *CEPŬLLA, ecc., non è difficile supporre che la -LL-, scempiandosi, non sia venuta a convergere con l'esito della - L- semplice, e che essa, oltre alla durata relativamente più lunga, avesse un'articolazione più forte o enfatica. ${ }^{26}$

2.4.13. In parolla, tolla, la $\mathrm{Q}$ dal dittongo secondario $\mathrm{AU}$ deve essersi

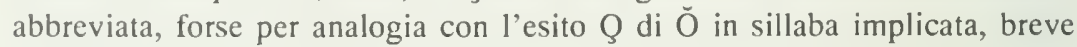
in OSS ǑSSU, vott ŎCTO. ${ }^{27}$

2.4.14. Analogamente a quello della liquida $l$ potrebbe spiegarsi il rafforzamento delle sorde. Anche qui le consonanti che, digradate, quindi soggette al dileguo, come generalmente le sonore originarie, vengono a trovarsi all'uscita in seguito alla caduta delle vocali finali, lasciano le toniche precedenti prolungate in sillaba originariamente libera: sēd SǏTE, nevōd NEPŌTE, 
crèd, rìd, fadigh, nās NASU, dès DĚCE, lḯs LUCE, cec. (Salvioni 1884: passim) (cfr. § 2.4.5.), mentre risultano brevi quelle seguite da consonanti sorde (che, quindi, si rafforzano), discendenti da doppie originarie o secondarie, o da gruppi consonantici pervenuti a sorde, ecc.: mèt MITTTERRE, nagott (U)NA + GŬTTA, vèss ESSĔRE, pass PASSU, sè t SĔPTE, rò t RŬPTU, sò SÜBTU(S), pèss PİSCE, biott got. blauts, strècé STRǏCTU, tèćć TECTU, nòćć NŎCTE, frèćć FRIG(I)DU, ecc. Anche qui il rafforzamento consonantico si sarà poi per analogia propagato in altra posizione (gotta, vacca), perfino dove si ha la sonora (veggia, oreggia, ecc., corrispondenti a vèćć, orèćć, ecc.: cfr. nota 19).

2.4.15. Per quanto riguarda le nasali, e il loro influsso sulle toniche precedenti, il milanese offre il seguente quadro: la $-n$ finale romanza nasalizza la tonica precedente, fondendosi con essa, che risulta lunga (e chiusa per i timbri $e$, o da tutte le basi): $m \bar{a}, p \bar{a}, s \bar{a} \mathrm{SANU}, b \bar{o}$, piē $\sqrt{\imath}, v \bar{u}$, ecc. (Salvioni 1884: passim), mentre breve (ed aperta) è la tonica precedente le nasali ( $n-$-, -m-) intervocaliche, anche quando, per ragioni morfologiche, o altre, vengono a trovarsi in posizione finale. ${ }^{28}$ L'allungamento, nel primo caso, sarà certamente da porsi in diretto rapporto con il dileguo della $-n$ finale, dietro la nasalizzazione, quindi assimilazione ad essa, della tonica precedente. Pare che la nasalizzazione si fosse affermata, anticamente, anche davanti a - $n$ intervocalica: nasalizzazione che si sarebbe successivamente perduta, come in francese (Rohlfs 1966: § 223). Non è possibile stabilire una cronologia esatta delle fasi successive che hanno portato ai risultati odierni: -on( $n) a$ da -ŌNA, -ŎNA, -en(n)a da -ĒNA, ${ }^{29}-i n(n) a$ da -İNA, - - $m n(n) a$ da $-\bar{U} N A,{ }^{30}$ -an(n)a da -ĀNA (coronna, bomma, cadenna, lanna, ecc. anche Romma) con la tonica precedente breve ed apertissima e $-n(n)(\mathrm{e}-m(m))$ pronunciate rafforzate, e perciò stesso rappresentate per lo più con la doppia. Trattando della - $m$ - originariamente intervocalica, il Rohlfs (1966: § 222) riconosce il fenomeno, peraltro assai diffuso in parecchie zone, fra gli altri dialetti per il milanese, citando famm 'fame,' pomm, fiümm, ecc. e dichiarando che " . . . in queste forme il suono in realtà non è oggi certamente da considerare rafforzato, però la brevità della vocale precedente permette di riconoscere che in altra epoca la consonante dev'essere stata lunga." Al medesimo fenomeno di abbreviamento vocalico che, come si è visto, si producc davanti a -nintervocalica non v'è alcun accenno. Se dell'abbreviamento vocalico è da tenersi responsabile il rafforzamento consonantico secondario, verificatosi nel passato nel caso della -m-, come sostiene il Rohlfs ncl brano citato, di un rafforzamento consonantico deve esscrsi pur trattato anche per la $-n-$. Le diverse fasi di questa evoluzione possono forse spiegarsi supponendo che, in seguito alla denasalizzazione davanti a -n- intervocalica (e finale corrispondente) (se anticamente anche la $-m$ - nasalizzassc la tonica precedente, non è possibile accertare), la $n$ (che forse non aveva mai cessato di essere pronunciata) c la $m$ si siano raffozzate in $-n n,-m m$ quando finali, scguendo la tendenza generale 
delle consonanti finali a consolidarsi (parallelamente a quella delle sonore a passare nelle sorde corrispondenti), provocando l'abbreviamento e l'apertura delle toniche precedenti. ${ }^{31} \mathrm{E}$ il rafforzamento consonantico, dalla posizione finale, deve essersi esteso a quella intervocalica, per cui bonna, cünna, finna, pienna, sanna accanto a bonn, cünn, finn, picnn, sann, ecc. (cfr. $\$ 2.2 .5$.). ${ }^{32}$

2.4.16. Per quanto riguarda la correlazione tra il grado d'apertura e la quantità delle vocali, il Salvioni (1975: § 5) nota: "La differenza tra vocale aperta e vocale chiusa si riduce in fondo, e ancor più si riduceva originariamente, a una differenza quantitativa in quanto aperto dica breve, e lungo dica chiuso. Ciò appar chiaro da ciò che, alla finale, un $e$ tonico lungo non possa esser che chiuso, e aperto un $e$ tonico breve . . ., e altrove (1911: 371, n.4): “ . . il milanese, data vocale lunga alla finale, la vuole chiusa, mentre la vuole aperta se breve (cfr. $k u u=k g$ capo, di altre parti della Lombardia)." Anche se il Salvioni parla qui più specificatamente della posizione finale uscente in vocale, e a questa posizione si riferiscono gli esempi da lui riportati nel passo qui sopra citato ad eccezione di eel? "è egli?" non è difficile ammettere che egli aveva in mente tutt'e due le posizioni di cui sopra (cfr. §2.4.1.). A questo riguardo si noterà, però, anche che, per $a, i$, $u[y], \ddot{o}$, in Fonetica non si trova alcun accenno a una variazione del timbro secondo la quantità. ${ }^{33}$

2.4.17. Da ciò che si legge in Salvioni (1884: 153) che "la posizione milanese intacca sempre più o meno la qualità della vocale cui essa segue: $\grave{e}$ per $i$ ed $e$, ò per $o, \zeta$ o un suono molto affine per ï." (cfr. § 2.4.9.), si sarebbe indotti a credere che la qualità aperta (e quindi la brevità) della tonica in "posizione" dipenda, o sia stata determinata dal rafforzamento della consonante. E così sarà stato in quei casi in cui la consonante rafforzata è preceduta da una tonica originariamente lunga e chiusa (vell VÉLU, venna VĒNA, remm RĒMU, padronna PATRŌNA, soll SŌLU, ecc.). Si può anche pensare che la tonica, ad es., di penna PĒNA, che il Salvioni (1911: 374) ritiene che fosse ancora chiusa ([e]) nel periodo di Bonvesin per aprirsi in $[\varepsilon]$ in un periodo successivo, attraverso uno sviluppo fonetico le cui modalità ci rimangono ignote, che questa $[\varepsilon]$, venendosi a trovare in sillaba libera, in cui già prevaleva [e] chiusa da tutte le basi $(\breve{\mathrm{E}}, \mathrm{AE}, \overline{\mathrm{E}}, \breve{I})$, sia stata sentita come di sillaba implicata e che ne sia conseguito il rafforzamento della consonante come in sillaba implicata (cfr. però, § 2.4.15.). Con questa ipotesi contrasta, però, il fatto che lo stesso fenomeno ci si presenta anche in soll SŌLU (forse anche in lof (loff in cherubini (1839: s.v.)), senza che vi fosse una [ग] aperta. Comunque si voglia spiegare tali forme, il rafforzamento consonantico si manifesta principalmente in quelle consonanti sorde che chiudevano, e chiudono, la sillaba: sett SĔPTE, secc SĭCCU, vott OCTO, rott RŬPTU, bocca BŬCCA, ecc. In tutti questi casi, pare che sia stata la brevità della tonica ${ }^{34}$ a provocare il rafforzamento della consonante susseguente, $o$ che la consonante preceduta da tonica breve si sia rafforzata per una specie di 
isocronismo sillabico, dato che alla tonica lunga faceva, per contro, seguito una consonante breve.

2.4.18. $\mathrm{Ci}$ si può domandare se i] rafforzamento si fosse compiuto già nel periodo di Bonvesin. Fenomeni collegati con esso sono, come si sa, la brevità e l'apertura della tonica precedente. Secondo la nuova norma che regolava la quantità delle vocali nel romanzo occidentale, erano divenute brevi le toniche in sillaba implicata (si veda nota 34). Il Salvioni (1911: 373) crede, in base alle rime presto / desco (N 13-4); erbeta / violeta / breta / asseta (<ASSĔDİTARE) (G 141-4); ecc. (Contini 1941: 82, 316) ${ }^{35}$ che in tale sede il riflesso unico di $\overline{\mathrm{E}}, \overline{\mathrm{I}}, \breve{\mathrm{E}}$ fosse $[\varepsilon]$. $\grave{\mathrm{E}}$ impossibile determinare se la consonante seguente la tonica, in questa ultima serie, avesse allora un'articolazione forte. Ricorre, però, la rima bella / cautella / apella / rebella (Q 245-8) (Contini 1941: 185). Il fatto che cautella CAUTÉLA rimi con voci che hanno una $[\varepsilon]$ potrebbe additare a una pronuncia aperta, forse d'origine dotta, della sua tonica. Si può, però, anche supporre che la pronuncia aperta della tonica fosse dovuta all'effetto della - $l$ - forte, analogica con -l(l)- da -LL(cfr. $\$ 2.4 .11$.). Breve ancora, ma, diversamente dal riflesso di $\overline{\mathrm{E}}, \overline{\mathrm{I}}$, $\check{\mathrm{E}}$, chiuso deve esser stato quello di $\overline{\mathrm{O}}, \overline{\mathrm{U}}$ in sillaba implicata, come crede il Salvioni (1911: 371) che fosse nella rima boca / negota (<(U)NA + GŬTTA) (N 147-8) (Contini 1941: 320), le cui toniche oggi si riflettono per [u] e [0] rispettivamente. 11 diverso esito in negota (cioè nagotta: Cherubini 1839: s.v.) egli spiega con “. . . una più generale tendenza che corresse parallela a quella per cui si ha męt mettere ecc.," e di cui rott, sott, ecc., insieme a negota, sarebbero i casi superstiti (Salvioni 1911: 373, n. 3). L'apertura di [o] in [0] in negota, ecc. sarebbe, dunque, secondo il Salvioni, da ascriversi all'influsso analogico della $[\varepsilon]$ in cui erano venuti a coincidere i riflessi di $\breve{E}, A E$ ed $\bar{E}, \breve{I}$ in sillaba implicata. In quei casi, che a tale influsso si sono sottratti, la [o] ha potuto chiudersi ulteriormente in [u] come oggi in bocca, coppa, ross, toss TŬSSE, ecc., ${ }^{36}$ che hanno la tonica breve, ma chiusa, contro la norma generale che la vuole, invece, aperta. La correlazione, valida per l'altra vocale media, per cui alla chiusura corrisponde una maggiore durata, c viceversa, risulta così, per quanto riguarda [u], in parte perturbata.

2.4.19. In Salvioni 1975, la parte dedicata alla fonetica milanese è concepita in modo del tutto diverso rispetto alla Fonetica (Salvioni 1884), in quanto basata su criteri comparativi, pratici, piuttosto che storici, e ha come termine di paragone l'italiano letterario (cfr. \$ 1: "All'a tonico dell'italiano, Milano corrisponde con ee ed $e \ldots$ "; $\S 7$ : "Non rari i casi, ne quali all' $e$ tonico (per lo più chiuso) italiano, è corrisposto con $i$. . .," ecc.). Anche se, in aggiunta alle forme proprie del Porta, molte ne vengono riportate dal Salvioni che gli sono estranee, questo lavoro pur rimane quello che sostanzialmente è: una guida per la lettura del Porta. E appunto perché una guida, il Salvioni non elabora, non specifica, non volendo appesantire il suo esposto con distinzioni minute del tipo di $n(n)$ né semplice né doppia, e si limita a dare norme gene- 
rali, quali (§35) "Al milanese mancano completamente le consonanti doppie, malgrado l'abuso che se ne fa invece nella scrittura.": norme che, per esser meglio intese, vanno integrate con quanto egli ci insegna nella sua Fonetica (Salvioni 1884) (e negli altri lavori suoi citati sopra).

2.5.1. Il sistema adottato da Isella (1959) è in massima quello ricavabile dal Salvioni (1975), di cui lo stesso Isella ha del resto curato l'edizione.

Anche per Isella (1959: 649) “ . . . la differenza di qualità è anche una differenza di quantità, poiché una vocale aperta è breve e una vocale lunga è chiusa.," come appare da pè 'piede,' mè 'mio, -a,' trè (forma del femminile), mò (MŎDO), tò 'tuo, -a' contro pee 'piedi,' mee 'miei, -e,' lee 'lei,' assee 'assai,' poo 'poco,' coo 'capo,' soo 'io so,' ecc. (eccezionalmente bèe 'pecora' (voce onomatopeica), eel? eela? (forma interrogativa del verbo essere)).

2.5.2. Nota ancora Isella (1959: 649) che "Si pronuncia grosso modo chiuso ogni $e$ tonico di sillaba attualmente o in origine uscente in vocale; aperto, invece, ogni $e$ di sillaba attualmente $o$ in origine uscente in consonante.," e dà gésa 'chiesa,' maltésa, pél 'pelo,' pés 'peso,' més 'mese,' contro nébbia, sèggia 'secchia,' pèll 'pelle,' pèss 'pesce,' fèrr (ma vérd, sérc 'cerchio,' vérz 'cavolo,' il nesso $r+$ cons. non facendo sempre posizione); che, in generale, alla [ग] dell'italiano letterario il milanese fa corrispondere la stessa vocale: còsta, nòcc, òca, pòcch, pròppi o pròpri (eccezionale il caso del nesso consonantico al + cons. che in milanese può riflettersi in ol: cold 'caldo,' solt 'salto,' olter 'altro,' ecc.: cfr. nota 27), mentre in corrispondenza della [o] (ma anche della [u]: lôff 'lupo,' ôff 'ufo,' ecc.) il milanese presenta [u]: fiór, onór, nós 'noce,' inósca, curiós, ecc.

2.5.3. Per le rimanenti vocali, la cui quantità non può inferirsi dal timbro, Isella (1959: 650) ci dà una norma empirica. Mentre per [a] [i] [y] la grafia normalmente distingue, in finale, la tonica lunga da quella breve, rappresentando la prima con la doppia (Inangiaa, vestii, riduu 'riso' (participio passato e sostantivo) contro mangià, vestì redù (infiniti) (e qui omette [ø], omissione che sembra sia dovuta al fatto che essa, a differenza di queste altre vocali, non viene mai scritta doppia: fioeı FILIÒLU), “ . . è lunga ogni vocale seguita da una sola consonante (oppure da $r r$ ), breve quella seguita da due consonanti o più.": pas 'pace,' cas 'caso,' tir 'tiro,' fil 'filo,' fus 'fuso,' mur 'muro,' carr 'carro' ma pass 'passo,' cass 'casse,' fiss, bestiolitt, fuss 'fossi, -e,' guss 'guscio,' ecc. ${ }^{37}$

Lunghe e chiuse, inoltre, le toniche davanti a $-n$ finale con cui si fondono, nasalizzandosi (pan, fen 'fieno,' ven 'viene,' vin, don 'dono,' bon, vun), e davanti a $n, m$ complicate in fin di parola (redond) mentre in fin di sillaba la nasalizzazione è meno forte (ongia 'unghia,' semper, zenta 'cinghia,' ecc.) (Isella 1959: 651) (cfr. nota 28).

Poiché con la -n scempia finale si suole indicare la nasalità della tonica 
precedente, $^{38}$ per denotare l'assenza di nasalità si fa ricorso alle grafie $11 m$, mm: pann 'panno,' cann 'canne,' fenn 'fateci' venn 'vene,' donn 'donne,' bonn 'buone,' fumm 'fumo,' ecc. La tonica, in questi casi, è breve c aperta (come anche davanti a $\bar{n}$ : pègn, sògn, ecc.). "Da qui il vezzo di scrivere $n n$ e $m m$, per indicare che si tratta di consonante dentale, anche all'interno della parola e fuori di uscita di sillaba. Esempi: veggianna, picnna, funna, bonna, ıumna ecc., accanto alle grafie veggiana, piena, finu, bona, iuna, ccc." (Isella 1959: 652).

Asserendo che "La pronuncia del milanese non conosce consonanti doppie, la grafia invece ne fa largo impiego, spesso per semplice uniformità alla grafia dell'italiano," Isella (1959: 650) sembra esludere del tutto il rafforzamento delle consonanti in certe posizioni, ma possono valere qui le stesse considerazioni che si sono fatte al $\S 2.4 .19$.

2.6.1. Beretta (1980: 9-10) distingue per il milanese i fonemi vocalici [a] [a], ${ }^{39}[\mathrm{e}][\varepsilon],[\emptyset][\mathrm{oe}],{ }^{40}[\mathrm{u}][\mathrm{o}],[\mathrm{i}],[\mathrm{y}]$, e pone, per ciascuno di essi, varie gradazioni che vanno dalle più aperte alle più chiuse (collegate alla loro relativa brevità e lunghezza rispettivamente), come nei seguenti esempi: [å] pas, magnan, andaa; [a] pass, matta; [e] almen, pel, ver, seda, andee, andeghee; [ع] vedè, pell, cadena, vers, bee; [ø] coeur, roeud, (i) floeu; [oe] (el) froeu, fioeula, voeuna, noeura; [u] nevoda, coo; [0] (i) bòtt, coll, l'or [y] tutt, vun, mul, mur, bevuda, bevuu; [i] fini, fin, fil, tir, partida, finii, ecc.

2.6.2. Sono lunghe (e chiuse), nel sistema Beretta, le toniche di sillaba libera, originariamente e attualmente penultima (fila, tosa, veder VITTRU), di sillaba divenuta ultima, uscente in consonante semplice (pas, pes, $f i l$ ), in $r$ + cons. (verd, serc), in nasale $-n$, in vocale doppia (andaa, dormii, veduu, coo CAPU(T)), in ô (resgiô RECTŌRE); lunghe (ma aperte) nelle sequenze -ona, -ena, -ina, -ana, -oeuna, -una (rana, pena, fina, dona, voeuna, luma) (e qui Beretta differisce, fra gli altri, dal Rajna (\$ 2.3.1.), dal Salvioni (§ 2.4.8.), da Nicoli ( $\$ 2.7 .4$.) per i quali la $-n$ - ha durata normale, pur presentando una maggiore intensità). D'altra parte brevi (ed aperte) le toniche seguite da consonante doppia (matt, pess, camn, famm), nelle desinenze degli infiniti (andà, vedè, guari); brevi ancora (ma con variazione di timbro) quelle di parole originariamente proparossitone (sèdes $\mathrm{SEDECI}(\mathrm{M})$, pòmis, rédus lóder. ecc.) (Beretta 1980: 18-19).

2.6.3. Beretta ammette, per il milanese, oltre ovviamente alle brevi, non solo consonanti lunghe (relativamente più lunghe dopo tonica (matta), meno lunghe dopo atona (mattocch)), ma anche le doppic, equivalenti alle italiane, ma solo in fin di parola (matt) (Beretta 1980: 22).

2.6.4. Dalla descrizione di Beretta non risulta del tutto chiaro il valore quantitativo delle toniche penultime. Dopo aver notato che "Hanno suono generalmente chiuso e prolungato" (Beretta 1980: 18) le toniche in fila, rosa, tosa, ecc., presenta come di media durata, anche se nella stessa configurazione 
sillabica di fila, quella di cala, pela (Beretta 1959: 31) perché susseguita da consonante di durata media (contro cal, pel da una parte, e call, pell dall'altra, in cui a vocale lunga e breve fanno seguito rispettivamente consonanti brevi e lunghe). Sembra, dunque, che Beretta consideri di media lunghezza le toniche penultime precedenti la liquida - $l$ - (fila, cala, pela), ma lunghe quelle precedenti le sonore in generale (rosa, tosa, veder, partida, bevuda, nevoda, seda) e, come si è visto sopra, anche le nasali -n-, -m- (cadena, bestema). Su questi ultimi punti, almeno, differisce Beretta dal Salvioni. Per questi la lunghezza vera e propria è quella che risiede in sillaba finale (cfr. § 2.4.3. e la nota 37 ), ed è meno lunga la tonica penultima, la cui quantità pare sia determinata solo dall'accento; brevissima quella delle sequenze -ina, -ena, ecc. (cfr. § 2.4.15.).

2.7.1. Secondo Nicoli (1983: 46), il sistema vocalico tonico milanese consta di nove fonemi vocalici ([å] [a], [e] [e], [u] [o], [ø], [y], [i]) che possono essere lunghi o brevi. La quantità delle vocali dipende dalla struttura sillabica: generalmente lunghe le vocali toniche seguite da consonante semplice, brevi quelle seguite da più consonanti (Nicoli 1983: 44) (con alcune eccezioni per $o[\mathrm{u}$ ], specie nei monosillabi rôss [rus] 'rosso,' sôll [sul] 'solo' (Nicoli 1983: 50, n.4). In tale posizione si ha correlazione di lunghezza chiusura per [e] (sempre), [å], [u] (sovente), e di brevità - apertura per [ $\varepsilon$, a, ग] rispettivamente, come nei seguenti esempi (Nicoli 1983: 45, 50):
pés [pe:z] 'peso'
pél [pe:I] 'pelo'
pas [på:z] 'pace'
pèss [pes] 'pesce'
pèll [pel] 'pelle'
pass [pas] 'passo'

(ma anche
car [kå:r] 'caro'
tôsa [tú:za] 'ragazza'
carr [kår] 'carro')
mòssa [mosa] 'mossa'

(ma anche

ròs [ro:z] 'le rose'

rôss [rus] 'rosso').

Anche in posizione finale libera, per [å] [a], [e:] [ع], [u:] [0], parallela alla distinzione di quantità, corre quella del grado d'apertura: andaa [andå:] 'andato' contro andà 'andare,' pee [pe:] 'piedi' contro pè [p'́] 'piede'; coo [ku:] contro tò, sò [to, so] 'tuo, suo.' Limitatamente a [y], [i], [ø], è possibile parlare di un'opposizione basata sulla sola quantità in sillaba finale implicata (fus [fy:z] 'fuso' / fuss [fys] 'fosse'; (la) vit 'vite' / (i) vitt 'le vite') e in sillaba finale libera (ridù 'ridurre' / riduu [ridy':] 'riso,' vesti 'vestire' / vestii [vesti:]; fioeu 'figlio' / fioeu 'figli,' ecc.) (Nicoli 1983: 45-46, 49).

$\mathrm{La}-n$ finale si dilegua nasalizzando la tonica precedente che risulta lunga: can [kã:] 'cane' contro cann [kan] 'canne,' pan [pã:] 'pane' contro pann [pan] 'panni.' Una nasalizzazione meno evidente si ha pure davanti a $n, m$ complicate (Nicoli 1983: 45, 56-9).

Per Nicoli, il milanese non possiede consonanti geminate quali esistono 
in italiano. "Esistono semmai consonanti lunghe, pronunciate con un intensità maggiore delle altre, soprattutto se si tratta di consonanti finali." (Nicoli 1983:48). Egli pare ammettere una certa intensità consonantica (minore rispetto a quella che si ha in fin di parola) per le nasali, le sorde e spesso per le liquide intervocaliche: bonna 'buona,' penna 'pena, penna'; lamma 'lama,' pippa 'pipa,' fiffa 'paura,' terra; colla, ecc. Le toniche penultime seguite da tali consonanti tendono ad essere (o sono) brevi cd aperte.

Nicoli non sembra fare alcuna differenziazione fra la quantità vocalica in posizione finale, c quella in sillaba penultima.

3.1. Dall'analisi precedente delle descrizioni del sistema fonetico milanese si può stabilire quanto segue. Le toniche paiono essersi allungate davanti a consonanti indebolite (le sorde digradate a sonore, le labiali sino a fricative, per farsi poi soggette alla caduta come le sonore originarie; la $-n$ finale che si dilegua lasciando nasalizzata la tonica precedente; $-l,-r$ finali labili (talvolta) in sillaba originariamente libera e parossitona. Esse risultano più lunghe in sillaba divenuta ossitona (uscente, o no, in consonante) in seguito alla caduta delle vocali finali romanze, meno lunghe in quella attualmente parossitona sed-seda, roeud-roueda, rav-rava, rar-rara, ar (al) -ara (ala), pan, san (ma samna), bon (ma bonna), pee 'piedi,' assee, abaa 'abate,' andaa 'andato,' ecc. Generalmente lunghe pure davanti al nesso $r+$ cons. (larg, larga), forse davanti a $n, m$ complicate in finale (temp, redond), davanti a $r$ (ferr), quelle che risultano dalla contrazione di nessi vocalici o di dittonghi secondari (coo CAPU(T), assee AD SATIS, ecc.). Brevi, invece, risultano le toniche in contesti diversi da quelli specificati sopra, come, ad es., per non parlare che dei principali, davanti a consonanti sorde (sell, secch, pess, rott, mutt "muto"; Ioss TŬSSE, ecc.), davanti a $n, m$, talvolta $l$ intervocaliche e finali (boma, sanna, bonn, sann, goll, golla, GULLA, fradell, ecc.), davanti a gn, e palatali in genere (anche sonore) (vecc, veggia, frecc, freggia, legn, ecc.) che, quasi per compensare la brevità della tonica precedente, vengono articolate con più forza, e, quindi, graficamente rese per lo più con la doppia.

3.2. Esiste una correlazione fra la quantità e il grado d'apertura per [e:] $[\varepsilon]$, in parte per $[u][\supset]$. Alcune descrizioni (Rajna, Beretta, Nicoli) riconoscono tale correlazione anche per [å:] [a], e (limitatamente a Beretta) per [ø:] [oe]. Altrimenti [ø:] [ø], e [y:] [y], [i:] [i] (ancora secondo Beretta relativamente più chiuse e più aperte a seconda della loro quantità).

3.3. Questi dati si possono ravvicinare ai risultati cui giunge $\mathbf{M}$. Durand nel suo importante lavoro sulla durata vocalica (Durand 1946). Fra le caratteristiche fonetiche, opponenti le vocali lunghe a quelle brevi, questa studiosa, oltre a intensità decrescente, tono discendente, una maggiore espirazione per le prime (e intensità crescente, tono montante, minore espirazione per le seconde), annovera una differenza nella tensione e nel taglio sillabico (Durand 1946: 162). 
3.4. Si distingue un taglio sillabico 'forte,' in cui la consonante sussegue al massimo d'intensità della vocale precedente, da non permettere a questa di realizzarsi pienamente, da quello 'debole,' in cui la consonante è congiunta con la vocale precedente dopo che questa ha avuto il suo pieno svolgimento. Anche se non si può affermare categoricamente che le vocali lunghe sono seguite da un taglio debole, e le brevi da un taglio forte, Durand è propensa a vedere qualche rapporto fra la durata lunga e breve e il taglio sillabico rispettivamente debole e forte (Durand 1946: 161).

3.5. Il contatto fra il fonema vocalico e quello consonantico nella voce milanese sed 'sete' è presumibilmente debole. D'altro lato, è forse possibile ravvisare un fenomeno simile al taglio (o contatto) forte nella cosiddetta "posizione milanese" (cfr. $\S \S 2.4 .8 ., 2.4 .9$.). E' vero che, nella definizione datane dal Rajna, si dice, della consonante che si trovi in tale posizione, che essa "... invece di ripartire le sue articolazioni tra la vocale antecedente e la seguente, le appoggia per intero alla seguente, quasi fosse scritto bo -nna.," mentre nel taglio forte la consonante intervocalica viene distribuita diversamente, annettendo parte della sua articolazione alla vocale precedente. Tuttavia, da quanto si legge nel Cherubini (1839) a proposito dello stesso fenomeno (cfr. §2.2.6.) sembra di potersi concludere che le consonanti intervocaliche facenti "posizione" debbano piuttosto considerarsi come parte della sillaba precedente.

3.6. $\quad \grave{E}$ noto che, in quanto articolate con minore energia, normalmente hanno carattere debole (lene) le consonanti sonore rispetto alle sorde corrispondenti, la cui articolazione, comportando uno sforzo (o tensione) muscolare di maggiore intensità, è, invece, forte e leggermente più lunga. Una consonante forte (sorda) esercita un influsso assimilatorio di tensione sulla vocale antecedente che viene, quindi, percepita come breve." In conformità di ciò, e secondo le conclusioni di Durand (1946: 165) "La même consonne est plus longue lorsqu'elle suit une brève que lorsqu'elle suit une longue ou une diphtongue" e che "La consonne précédée d'une voyelle longue est moins forte que celle qui est précédéc d'une brève." Si avrebbe, dunque, una specie di isocronismo o equilibrio sillabico, per cui a una consonante debole corrisponderebbe una vocale lunga precedente, e una vocale breve a una consonante forte. La quantità di una vocale verrebbe, così, ad essere inversamente proporzionale all'azione assimilatrice della consonante seguente. Secondo Durand (1946: 173), inoltre, le consonanti deboli (allunganti) e forti (abbrevianti) non sarebbero necessariamente le stesse da una lingua all'altra. ${ }^{41}$

3.7. Lo stesso equilibrio sillabico si osserva anche nel milanese. Anche in questo dialetto, infatti, le vocali lunghe sono seguite da consonanti originariamente sonore, leni, in alcuni casi del tutto eliminate a causa della loro debolezza stessa, e le brevi dalle sorde, per lo più provenienti dalle doppie originarie (cfr. §3.1.). È vero, come si è notato (cfr. 2.4.6. e la nota 46), che 
alcune delle sonore sono oggi desonorizzate, la tonica antecedente rimanendo sempre lunga (noeui, sed). In tal caso si dirà che la consonante, pur perdendo la sua sonorità, ha mantenuto il suo carattere debole. Anche la liquida $l$ ha varianti deboli e forti. ${ }^{42}$ Come si è notato in precedenza (cfr. $\$ \S 2.4 .10$., 2.4.11.), la $l$ pare aver avuto carattere debole in antico milanese, poiché si è conguagliata con $r$ in parecchi casi, quando intervocalica, e quando finale, dopo tonica, si è dileguata. La $l$ finale, oltre a questa articolazione debole (sonora), per cui tendeva ad ammutolirsi lasciando la tonica precedente lunga (saa, maa, cugiaa), deve averne conosciuta un'altra, relativamente più forte, forse sorta per analogia con $-l$ discendente dalla -LL-geminata (pell PELLE) che avrà provocato l'abbreviamento della tonica precedente per dare mull MŪLU, soll SŌLU, vell VĒLU, ecc.

3.8. Di un'articolazione debole è il caso di parlare anche per la -r finale, per cui comune è in milanese la sua caduta nelle voci parossitone originarie (salve quelle ridotte a monosillabi: rar, car) e in tutti gli infiniti, compresi quelli della $11 l$ coniug. (mett). Eccezion fatta per gli infiniti (parlà, senti), la tonica precedente questa $r$ debole, ammutolita o meno, è lunga. Lunga, però, risulta oggi anche la tonica precedente $-r r$ (-RR-) in carr, ferr (con [ $\varepsilon:])$, corr, torr, meno lunga in terra. Sarà interessante notare poi che il nesso $r+$ cons. in milanese non fa sempre posizione, dato che il dialetto ci offre verd VĨRĪDE, verz VǏRĪDIA, serc CĬRCŬLU con la tonica chiusa e lunga, mentre in sillaba implicata normalmente si ha $[\varepsilon]$ da tutte le basi, come in vert (A)PĚRTU, perd PERDERE. Tutto ciò fa presumere che la $r$ milanese avesse originariamente carattere debole, ossia che la differenza fra la $r$ debole (soggetta all'assorbimento da parte della tonica precedente) e quella proveniente dall -RR-geminata non fosse notevole. ${ }^{43}$

3.9. Una specie di lenizione si dica pure il dileguo della $-n$ finale romanza, che lascia la tonica precedente nasalizzata e lunga. Per i diversi esiti davanti a nasali, si rimanda il lettore al $\S 2.4 .15$.

4.1. Ciò che emerge dalle descrizioni precedenti si riassumerà dicendo che il sistema vocalico tonico milanese comprende i seguenti fonemi vocali-

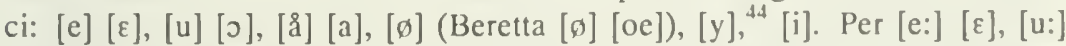
[ว], [å:] [a] la quantità è associata al grado d'apertura, essendo generalmente chiusa la lunga, aperta la breve. Costituirebbero, perciò, casi eccezionali lc vocali aperte e lunghe in pòc 'poco,' ćòd 'chiodo,' pòl, pòla 'può egli, può ella,' ćòs CLAUSU (con finale sonora accanto a ćòss dalla stessa base: Salvioni 1884: 293), fèr (ferr) FERRU presso il Salvioni (1884: 37, 293, 161), ròs 'le rose' presso Nicoli (1983: 45). Casi eccezionali sarebbero pure le vocali chiuse c brevi in toss presso il Biffi (Lepschy 1978: 197), sét (selt) 'sei o sai tu' presso il Salvioni (1884: 159, 278), rôss 'rosso,' gabaré, gilé presso Nicoli (1983: 45, 38) (ma gabarè, gilè presso il Salvioni (1884: 58; 1975: $\$ 5){ }^{45}$ 
4.2. In sillaba finale tonica uscente in consonante, le toniche sono lunghe davanti a consonante semplice originariamente (in taluni casi anche attualmente) sonora $^{46}$ (cfr. $\left.§ \S 2.4 .5 ., 2.4 .6 ., 2.4 .14.\right)$, davanti a $-n$ (nasalizzante) (cfr. § 2.4.15.), e talvolta liquida (cfr. $§ \S 2.4 .10 ., 2.4 .11 ., 2.4 .12$.$) . D’altra$ parte, davanti a consonante sorda (cfr. $\S \S 2.4 .8 ., 2.4 .9 ., 2.4 .14$.), nasale dentale (cfr. $\S 2.4 .15$.) o palatali in genere, talvolta liquida (cfr. $\S \S 2.4 .10 ., 2.4 .11$., 2.4.12) sono esse brevi ed aperte. Quasi tutte le descrizioni del sistema fonetico milanese che sono state qui sopra analizzate concordano nel considerare queste ultime consonanti rafforzate. ${ }^{47}$

4.3. Non è lecito parlare, in questa posizione, di una opposizione fonologica basata sulla quantità vocalica, ${ }^{48}$ trattandosi, nei seguenti casi, di contesti non identici, in cui si oppongono

i.) consonante sonora - consonante sorda (e rafforzata): per [å:] [a] pas 'pace' - pass 'passo,' cas 'caso' - cass 'casse'; per [e:] [E] pes 'peso' pess 'pesce,' mes 'mese' - mess 'le messe, ges 'chiese' - gess 'gesso'; per [u:] [u] tôs 'ragazzo' - toss 'tosse'; ${ }^{9}$ per [y:] [y] fus 'fuso' - fuss 'fosse'; per [i:] [i] lis 'logoro' - lisc 'liscio'; per [ø:] [ø] (Beretta [ø:] [oe] mancano esempi);

ii.) vocale nasalizzata - orale: pan 'pane' - pann 'panno,' can 'cane' cann 'canne,' san 'sano' - sann 'sane' (ma anche san 'sanno': cfr. nota 31); fin 'sottile' - finn 'sottili'; ven 'viene' - venn 'vene,' fen 'fieno' fenn 'fateci'; bon 'buono' - bonn 'buone,' don 'dono' - donn 'donne, ecc.

iii.) Si hanno, poi, coppie minime con l'opposizione [e:] [E] pel 'pelo' - pell 'pelle,' sed 'sete' - sett 'sette'; [u:] [o] vot 'voto' - vott 'otto'; [å:] [a] cal 'calo' - call 'callo,' (ma anche car 'caro' - carr 'carro' che Nicoli (1983: 45) trascrive, però, sempre con [å]; [i:] [i] did 'dito' - ditt 'detto,' ecc. Si ricordi, però, che essendo le variazioni di quantità accompagnate da quelle del grado d'apertura per [e:] [E], [u:] [o], [a:] [a] (per Beretta [ø:] [oe]) (secondo Beretta, poi, anche per le rimanenti vocali [i], [y] si hanno gradazioni più chiuse e più aperte a seconda della quantità rispettivamente lunga e breve), rimane sempre il problema di decidere quale dei due tratti, la quantità o il grado d'apertura, sia veramente rilevante nella coscienza di chi parla. Non è, tuttavia, difficile sostenere che, ad essere fonologicamente pertinente, sia il grado d'apertura e non la quantità, restando le toniche [e:] [u:] [å:] di pel, sed, vot, cal, anche quando le si pronunci brevi, sempre distinte da quelle ([E] [o] [a]) di pell, sett, vott, call in base al loro grado d'apertura. Limitatamente ad [i:] [i] (did 'dito' - ditt 'detto'), forse [y:] [y], [ø:] [ø] (per cui mancano esempi) è lecito parlare di una opposizione fonologica basata principalmente sulla quantità vocalica.

4.4. Lo stesso potrà dirsi per quanto riguarda la posizione finale tonica 
libera. Anche qui la quantità sarà distintiva solo per [i:] [i], [y:] [y], [ø:] [o] (fuii part. pass - fini inf.; riduu 'riso' - ridic 'ridurre'; fioen 'figli' - fiocu 'figlio'), essendo decisivo il grado d'apertura, e non la quantilà vocalica, per

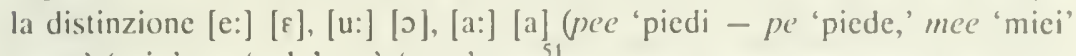
- mè 'mio'; su 'sole' - sò 'suo,' ecc.

\section{University of Guelph}

\section{NOTE}

1 II passaggio viene spicgato in vari modi, fra gli altri, da Lausberg (1947; 1972: §§ 155-6) in parte con l'influsso del sostrato osco-umbro; da Haudricourt-Juilland $\left(1970_{2}\right.$ : $31-42$ ) (c similmente da Lüdtke 1956) con la monottongazione in $\bar{e}$ del dittongo $\mathrm{AE}$, che avrebbe provocato uno squilibrio nel sistema vocalico latino, introducendo una nuova correlazione fra $\bar{e}<\mathrm{AE}$ e $\check{e}<\overline{\mathrm{E}}$ da una parte, ed $\bar{e}<\overline{\mathrm{E}}$ e $\grave{c}<\breve{I}$ dall'altra; da Weinrich (si veda più avanti nel testo); da Spence (1972) con la correlazione con $\bar{E}, \bar{O}$ da una parte e $\breve{E}$, Ŏ dall'altra delle varianti $\breve{\mathrm{E}}, \mathrm{O}$, inizialmente fonetiche, di $\check{\mathrm{I}}, \breve{\mathrm{U}}$, attestate nelle iscrizioni pompeiane, in rapporto di opposizione quantitativa con le prime, qualitativa con le seconde; si vedano, inoltre, Straka (1959: 286-300); recentemente anche Klausenburger (1975). Per un riassunto e discussione delle teorie accennate, si veda Tekavčić (1972: 15-33); Spence (1972: 300-9).

2 Cfr. Weinrich (19692: 183): “Unter besonderen Bedingungen kann es dabei vorkommen, wie wir oben an dem Wort tela gezeigt haben, daß der phonetische Anschluß weiter zurückreicht zu den phonologischen Vokalquantitäten des Lateinischen, aber nur in den Fällen, wo die lateinische Länge in freier, die lateinische Kürze in gedeckter Stellung stand: von dem phonologischen Vokalquantitäten des Lateinischen her gesehen ein Zufall."

3 Cfr. Weinrich $\left(1969_{2}\right.$ : 13): "Dic phonologischen Vokalquantitäten des Lateinischen sind überhaupt nirgends erhalten. Wo es heute in der Romania phonologisch relevante Vokalquantitäten gibt, z.B. im Friaulischen, sind diese sekundär enstanden."

4 Ha ragione il Salvioni (1919: 529-30) nel sostenere che il Biffi, con i termini 'largh' e 'strec' intendesse indicare una differenza quantitativa, visto che gli stessi termini vengono da lui adoperati per differenziare la tonica di dis 'dice' da quello di diss 'disse,' fra cui non corre alcuna differenza qualitativa. Una differenza qualitativa, oltreché quantitativa, si ha, invece, fra la $e$ 'strecia' in des, pel (oggi con [e:] lunga e chiusa) e la $e$ 'larga' in pess, capell (oggi con $[\varepsilon]$ breve e aperta) (a proposito della quale si legge che “. . . al à el son più visin a l'a ..." (Lepschy 1978: 192). Trattando, però, della $o[u]$, c non riuscendogli chiara la correlazione sia di quantità che di qualità, valida, come per l'altra, anche per questa vocale media, come esempio della o 'strecia' il Biffi riporta porscicl, la cui $o[u]$ è bensì chiusa, ma atona, quindi breve. Parimenti di una differenza qualitativa, accanto a quella quantitativa, doveva pur trattarsi per a a giudicare da quanto si legge a proposito: "Se proferiss a do foùsg, voùna largha e voùna strecia, e questa $\mathrm{i}$ Latin antighament ghe discuenn l'a longha c's la schriueuenn dobia insci amaabam, o con sora ona tireta $\hat{a}$, nun la parnonziem senza desconscià la bocha, come pas, nas, ... ghauasg; l'oltra slarghandela ben, come ma, la, à ..." (Lepschy 1978: 189-90).

5 “. . . parché no proferissem mai do consonant come $i$ Toschan, ma nomà voùna semper, e se ben enn schriuem do spess, al c̀ par fà cognoss che "I son và parnonzià più asper come dircm a la letera l." (Lepschy 1978: 191). La l, preceduta da vocale "larga,' "se parnonzia ghaiàrda" (Lepschy 1978: 195). Si noti che il Biffi non sempre sa distinguere la posizione finale uscente in consonante da quella assoluta, come quando afferma che "I temp futur che $i$ Toschan fenissenn in o largh, come potrò, vorrò, dirò, farò, ai le parnonzienn con la 
consonant aspra; e nun che i fenissem semper con l'o strec, i proferissem con la consonant dolza ..." (Lepschy 1978: 197).

6 Che la $o(<\overline{\mathrm{O}}, \breve{\mathrm{U}})$ rappresentasse [u], lo si deduce da quanto il Biffi afferma a proposito della [y] (cfr. nota 36), e dal fatto che egli spiega la tonica di toss come la diretta continuazione di quella latina in TŬSSE (senza tener conto, cioè, dell'evoluzione $\breve{U}>0$ ).

7 II Prissian non parla esplicitamente di un suono [y], ma il modo in cui descrive [ø] non lascia dubbi al riguardo: “. . où , che fa el son tra l'o largh e l'u vochà, ma più press a l'o; a dil se sporsg inn foùra i lauer de la bocha come a dì $u$, ma se slarghenn poù ben come a dì $o$ largh ..." (Lepschy 1978: 200).

8 Anche se il Prissian non lo dice esplicitamente, ci doveva pur essere una differenza di quantità (e di qualità) fra la tonica di choùr e quella di voùna: lo si deduce facilmente da quanto si legge a proposito della $n$ : “. . . par desferenzià pan da mangià da pann da vestiss, la proùma che se proferiss meza morta con la vochà strecia, la schriuarem sempia, l'oltra ch'è ghaiàrda con la vochà larga la schriuarem dobia ..." (Lepschy 1978: 196). Qui si parla più specificamente della $a$, ma il ragionamento doveva senza dubbio valere per tutte le vocali. Per la tonica di voùna, si veda la nota 16.

9 Dalla descrizione del Prissian non risulta una correlazione di quantità per [y] e [ø]. Per quanto concerne [y] deve trattarsi di una svista; per [ø], cfr. la nota precedente.

10 Secondo il Rajna (1881: 11), le tre varietà corrisponderebbero ai tre gradi di lunghezza: breve, media, e lunga.

11 A questi gradi, il Cherubini ne aggiunge poi un altro, quello rimesso, che forse qui sta per atono.

12 Non affatto chiaro risulta il rapporto fra la $o$ chiusa in dolór, onór, e quella chiusa e vibrata di sô (e di messô MİSSŌRIU, resô RASŌRIU, ordiô < ORDİRE). Se ıibrato equivale breve (e in questa accezione pare sia adoperato per $a, i, u[y]$ ), la descrizione del Cherubini contrasta con quella del Salvioni che cita $s o ̈$ con [0:] (anche messō, resō ordijō (1884: $\S$ 41 ), e poi, per quanto concerne le parole uscenti in -or (dolor, amor, ecc. dice (1884: § 34 nota) " . . I'ó è bensì lungo, meno però che in quelli dove -ore-si riduce ad $\bar{o}$."

13 Che -oo rappresenti sempre [o:], lo deduco da quanto dice al proposito: "Nè si creda che questo doppio $o$, trovato nel contesto del libro senza l'accento che gli si appone nella sede alfabetica, possa essere letto con quello sdoppiamento di vocale che sentesi nelle voci italiane Eoo, Coo, Coordinato, ecc., giacché abituati come siamo a trovare quella sdoppiatura italiana solo nell'o aperta, e a fare quel prolungamento di suono milanese soltanto nell'o chiusa ... " (Cherubini 1839: XXVIII o 2243).

14 "San (sano), Sann (sane), San (sanno), ccco tre suoni affatto diversi" (Cherubini 1839: 2246). Il Cherubini sembra qui distinguere fra la $-n$ dentale in san 'sanno,' la $-n n$ enfatica o forte in sann 'sane,' e la $-n$, non pronunciata, che nasalizza la vocale precedente in san 'sano' (cfr. nota 31).

15 "ö è il suono francese in feu, coeur ..." (Salvioni 1884: 36). Dagli esempi citati non consta se il milanese abbia una differenza qualitativa per oeu (eu), come, invece, ha il francese fra feu con $[\emptyset]$ e coeur con [oe].

16 "Non è tutt'affatto, ma vi si ravvicina l'u francese in un, commun, ecc." (Salvioni 1884: 35). Potrebbe, quindi, stare per una $\ddot{u}$ aperta (e breve) ([Y]): cfr. nota 30.

17 II Salvioni, che per questo suono adotta il simbolo $o$, dice prima che esso è l'italiano $o$ in Roma (1884: 35), che "Lo schictto suono di $u$ non esiste in milanese, e l'u che trovasi generalmente nelle scritture sta per $\ddot{u}$. Gli $u$ latini si ripercuotono in milanese parte per $\ddot{u}$ parte per $o$. Quest' $a$ (che in alcune scritture vien reso alla francese per $o u$ ) è per certo molto profondo, tanto da essere più vicino ad u toscano che ad ó." (1884: 36), aggiungendo in nota che "Nelle poesie volanti e nelle bosinād, cioc̀ nella letteratura del popolino il quale più che a convenzioni ortografiche obbedisce al proprio istinto acustico è frequentissima la grafia u per $o$. Da non molte di queste poesie estraggo: rispund « risponde " fuu $=f o \tilde{z}$ "faccio » sura $=$ sora «sopra $»$ discur $\ll$ discorrere $»$ desmunta spunta, stu = sto (isto) . . ." Lo stesso 
Salvioni dice poi, altrove, che " . . il dialetto milanesc, c già fin dai tempi del Porta, non conosce in realtà che un $o$, l'aperto. Il chiuso ì conservato solo nella scrittura, ma c, nella realtà della pronuncia, un vero c proprio u. . . " (1975: 6): cfr., invece, Lepschy 1978: 207, nota 39.

IS Al femminile -ada, -uda, -ida, col rafforzamento della fricativa interdentale di -adha, -udha, -illia (stadio prevalente nei testi antichi lombardi), probabilmente a causa della presenza di una $a$ finale ben salda (secondo il Rohlfs (1966: $\$ 203$ ) “ . . per ragioni di distinzioni del generc."), o forse per il ripristino della occlusiva nci riflessi -aa, -ruu, -ii, reperibili nei testi meno recenti (Salvioni 1884: § 379).

19 Omette però, il Salvioni, di precisarc che il prolungamento della tonica, nelle condizioni di cui sopra, si produce, a quanto pare, solo in sillaba libera (c davanti a certi gruppi di consonanti, come $r$ complicata), mentre brevi risultano le toniche, ad es., di spece SPEC(Ŭ)LU, ocucc OC(Ŭ)LU, ccc., perché, appunto, in sillaba implicata, anche se susseguitc da consonante originariamente sonora (spegio, ogio, oreg(i)e in Bonvesin, con affricata prepalatale sonora e vocal finale caduca).

20 In realtà con [ö:] lunga, come nota poi il Salvioni (1884: § 328, n. 1), correggendosi, per via di $i$ da -CT- (ERUCTARE), la qual vocalc lunga spicgherebbe la grafia roeud, registrata dal Cherubini (1839: s.v.) con-d (pronuncia sorda) (cfr., invece, Lepschy 1978: 208).

21 Per contro, dopo vocale breve si scrive la sorda anche quando corrisponda a sonora interna: vèć (accanto a '‘̀̀gga) VEC'LU, frèć (accanto a frèg'ga) FRİGīDU, ccc., e a trasgredire tale convenzione sarebbero goeubb, robb (plur. di robba) con $-b b$ a dispetto delle toniche brevi (Salvioni 1884: 160).

22 Anomalo il caso di loff 'lupo' come quello di criif 'crudo,' riif 'spazzatura' (Salvioni 1919: $536)$, con la tonica breve, per la cui -ff si veda Salvioni 1184: 160: "Faremo eccezione solo per $v$ d'uscita ridotto a $f$."

23 Pur riconoscendo il rafforzamento di certe consonanti nel milanese, il Salvioni sembra non essere d'accordo con l'uso di rappresentare graficamente consimili consonanti con le doppie che, come del resto tutti i dialetti settentionali, il milancse non conosce. Per un fenomeno molto simile di rafforzamento consonantico in alcuni dialetti connesi, si cita la seguente nota che si legge nel Rohlfs (1966: § 229, n. 1): “La conservazione delle consonanti doppie si può osscrvare nell'estrema fascia periferica dell'ltalia settentrionale. Essa è limitata alla posizione seguente la vocale accentata: cfr. nella Valscsia fussi 'tu fossi, 'navatta 'navetta,' cigulla 'cipolla,' bèlla, bukka, spassa 'spessa,' wakka (sccondo Spocrri); in alcune località della val Bregaglia gatta, bassa, štóppa, pénna, vacca (Stampa, 133). Non è ancora chiaro sc in queste zone si tratti di una tradizione continuativa, ovvero se si sia pervenuti ad un nuovo allungamento delle consonanti che seguono la sillaba breve accentata, a causa dell'accento; per questa seconda ipotesi sta il fatto che in Valsesia l'allungamento non si verifica dopo vocale lunga (per esempio péla <paela 'padella') c inoltre che qui anche la $m$ semplice compare in forma allungata: per esempio pummi 'pomi,' ommu, famme 'fame,' limma (Spocrri, 688); anche la pronuncia genovese di 'lima,' 'fame' e 'remo,' ciod limma, famme, remmu (cfr. AGI 16, 154), come pure il piemontese limma e in Valsesia lamma, pummi 'pomi' fanno pensare ad un allungamento secondario. ..." Si veda anche Bertoni (1916: § 42).

$24 \mathrm{E}$ ancora tèra (Salvioni 1884: § 204), la cui tonica deve considerarsi lunga, conformemente al sistema adottato dal Salvioni (1884: $160-1$, n. 2): “ . . Per $r$ ls ritengasi lunga la tonica precedente quando non sono scritte doppic. ..." Interessante l'osservazione del Rohlfs (1966: § 238): "Per quello che riguarda l'Italia settentrionale, la lunghezza della vocale che precede la doppia $r r$ di un tempo (per esempio in milanese fêr 'ferro,' tēra, kär 'carro') fa presumere l'appartenenza del passaggio di těrra a tëra ad un'epoca diversa da quella del fenomeno generale di degeminazione delle consonanti nell'italia settentionale, cioc̀ ad epoca più recente. ..."

25 In pedü (anche padii) (cfr. § 2.4.3) la tonica si sarà abbreviata dopo essersi allungata davanti a $-l$ finalc caduca. 
26 Di una tale articolazione forte e leggermente lunga della $-l$ in antico milanese potrebbe fornire un indizio il fatto che, mentre le geminate latine compaiono, nei testi di Bonvesin, normalmente ridotte alle scempie corrispondenti (accanto a grafie collatcrali latineggianti con la doppia), la grafia manticne $l l$ (castellan, collegia COLLECTA, coll CǑLLU, bella, apella, ecc.) non solo, ma l'introduce anche là dove non ha ragioni etimologiche (golla GŬLA, revellao REVELATU, parolla, ecc.; cfr. Salvioni 1884: 158).

27 È noto che, dietro la vocalizzazione della L e la conseguente riduzione del dittongo secondario $\mathrm{AU}$ che si era venuto a formare, il nesso $\mathrm{AL}+$ dentale, accanto ad altre varianti, ha dato $o l$, con una $l$ ipercorretta, frequente nei testi medievali lombardi (Bonvesin ha olio, oltro, coldo, ecc.). Questa $l$ ipercorretta compare, però, anche là dove si aveva originariamente AU: golzo GAUDIU, olzo AUDIO, golte (plur.) GAUTA in Bonvesin, el volsa *AUSA(T) (Salvioni 1884: § 63ß; Rohlfs 1966: § 42). Non è impossibile ravvisare tracce di una $l$ ipercorretta anche in parolla, tolla PARA(B)ŬLA, TA(B)UัLA.

28 Si può tralasciare qui, perché non di primario interesse, il trattamento della tonica davanti a nasale complicata. Basti precisare che, per i gradi medi di apertura almeno, si tratta di una vocale chiusa (e leggermente nassalizzata): témp, tént, frónt, mónt (Cherubini 1839: s.v.) (cfr. Salvioni 1884: $\S \S 15,41$ ). Non è chiaro su che cosa si basi il Rohlfs che invece cita, per il milanese, vęnt, ı̧mp (1966: § 98).

29 II Salvioni (1911: 370-1, 373-4) ritiene che le toniche nelle sequenze -cna, -ona fossero ancora chiuse al tempo di Bonvesin. Ciò egli deduce dal fatto che in rima o assonanza con le vocali chiuse (e nasalizzate) si presentano le toniche di ven, cren, pon, scarpon (seren I seren / ven, conven (G 73-6), sosten / ben / cren / ben (M 45-8); lomentason / cason / pon / devotion (E, F 301-4); scarpon / compassion / contrition / condition (O 213-6) (Contini 1941: 79, 233, 66, 285), forme abbreviate, secondo il Salvioni, procedenti da venno, crenno, ponno, scarponno (cfr. anche Salvioni 1919: 528, n.1). Egli sembra sostenere, dunque, che le toniche di ven, cren, pon, scarpon fossero tutte chiuse, senza, però, essere nasalizzate, in quanto, appunto, precedenti una $-n$ dentale da $-n n(o)$, non nasalizzante, come quelle delle sequenze -ena, -ona, ecc. Sarebbe, però, altrettanto lecito supporre che alla base ci fosse $-n(o)$. Tracce di una antica nasalizzazione forse sono, infatti, le vocali lunghe in han, in, ecc. del dialetio odierno, c la chiusura vocalica che si osserva in questa ultima forma. Una prova ulteriore di un'antica nasalizzazione, inoltre, è forse il fatto che essa compare in sinizesi, una volta, in Bonvesin, per cui Contini (1941: 251) scrive: De quii k'i in matremonio (B 396).

30 Pare che, nel passato, -INA si riflettesse per -enna (quaicosorenna, fiorentenna nel Prissian) e -ŪNA per -önna. Di tale stadio il dialetto odicrno non conserva che toeunna (cfr. Salvioni 1884: $\S 60$ ). Un fenomeno analogo, ancor oggi vivo nci dialetti emiliano-romagnoli, in epoca più antica abbracciava, a quanto pare, vasti territori della Lombardia (Rohlfs 1966: $\S \S 30$, 38). Si badi, però, che nei suddetti dialetti, l’apertura delle vocali è in dirctto rapporto con la loro nasalizzazione che, invece, produce vocali chiuse nel milanesc. Inoltre, all'apertura vocalica davanti a nasale fa riscontro, in quei dialetti, anche quella davanti ad altre consonanti

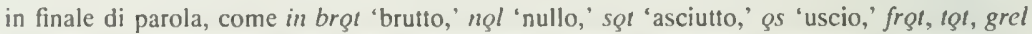
'grillo,' senkiu, maré 'marito,' aı'ó 'avuto, ecc. ai quali il milanese fa invece corrispondere brutt, cinqu, ecc. in cui tale passaggio non si verifica.

31 Lepschy (1965: 169, n. 36; 1978: 206, n. 36) dichiara: "Non so come si debba interpretare l'osservazione del Cherubini che si hanno tre suoni per -n finale come in san «sano", san "sanno», sann «sane»" (cfr. nota 14). La discussione precedente avrà in parte già fornito una risposta a questa domanda. In san 'sano,' si ha una [ā] nasalizzata allungatasi in seguito al dilcguo della $-n$ (il Salvioni (1911: 370) trascrive bulu, resúl m ma poi nota: "Scrivo così; ma rimane inteso che a Milano il - jè sostituito dallo strascico nasale della precedente vocale"; lo stesso Salvioni (1884: § 245) trascive $b \bar{o}$, sã , c dice della $-n$ che " . . si fonde . . colla vocale accentata precedente in una vocale nasalizzata ... "; cfr. anche Nicoli (1983: 56-8)). In san 'sanno' si ha una $-n$ dentale, non, però, soggetta al rafforzamento consonantico. Il Salvioni (1975: 42-3) scrive hin 'sono,' han, ma in Fonetica (1884: § 247) vann, fann, 
sann, ann, dann, inn, ecc. Li doppia $-n ! n$, in questo caso, starà solo per denotare la dentalità delia - $n$ (cfr. Isella (1959: 652), poiché la $-n$ scempia finalc indica, come si c̀ visto testè, la nasalità della tonica precedentc. Pare che oggi prevalga la grafia -nn (cfr. Beretta 1980: 129, 133; Nicoli 1983: 310, 11) che, invece, è di norma in sann 'sane,' come si rileva, del resto, dalla tonica lunga in san 'sanno' e brevissima in samn 'sanc."

32 Un esame minuto della rima nei volgari bonvesiniani ha consentito al Salvioni di concludere

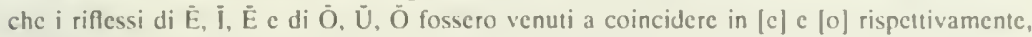
nel milanesc di Bonvesin, davanti a $-n$ finalc (Salvioni 1911: 370-1; 373-4) c che la nasalizzazione fossc già perfetta (Salvioni 1911: 387-8) (cfr. Contini 1935: 247; 1941: XXIX); nasalizzazione che egli pare ammettere non solo in bon BONU, ma anche in bon BONI, BONAE, data la rima qualc ad cs. bon / perdition / (angci) bon / perdition (A 269-72) (cui si aggiunga (l'orre) ... bon / intention / attention / saliation (T 493-6) (in Contini 1941: 39, 121), e, in base a prove metriche quale ad es. la sinizesi, in ceres maren $e$ galfion (T 245) (che, però, Contini (1941: 21) scrive ceres, maren, galfion), in marcn (o marcnc) $(<(\mathrm{A}) \mathrm{MARINA})$ che " . . . nun potrebbe valere che ncl supposto che il suo -n (sfumato poi nella nasalità della precedente vocale) sia un $-n$ gutturale ..." (Salvioni 1911: 387, n. 2). La nasalità della tonica egli sembra escludere nelle desinenze verbali -en, -on ( $3^{3}$ p. plur.) (Salvioni 1911: 388; cfr. però, nota 29), e nelle sequenze -ena -ona (Salvioni 1911: 374, ก. 1; 370, л. 4). Ne consegue che, secondo il Salvioni, la dentalità della $n n$, nel dialetto odierno, ad es. in bonn 'buone' c̀ per estensione analogica da quella intervocalica in bonna (Salvioni 1911: 387, n. 2; 1884: § 245). Con tutto ciò, non c̀ chiaro perché si sia prodotto l'abbreviamento (c apertura) della tonica precedente.

33 Che per [a] il Salvioni non ammettesse una variazione di timbro a seconda della quantità, risulta da quanto scrive (1919: 529), commentando un passo del Prissian (si veda qui la nota 4): “. . . dell'a . . . ammette duc pronuncie, una larga (cioc̀ breve) e una stretta (cioc̀ lunga), corroborata la prima dagli es. sarà salare, serrare, futuro di 'esserc' (oggi sará), la seconda da sarà salato, serrato (oggi sară . . E che il Prissian col suo 'largo' e 'stretto' intenda proprio indicare una differenza quantitativa, risulterebbe dagli analoghi termini ch'egli adopera p. es., parlando dell' $i$, ch'c̀ giudicato stretto in dis (oggi dis) 'dice,' c largo in diss (che oggi sarebbe indubbiamente dis; cfr. fis fisso, ecc.) 'disse, pur non correndo qualitativamente nessun divario tra $\mathrm{i}$ due $i$. ..." Per [ø], si veda la nota 15.

34 Una volta perduta la quantità fonologicamente rilevante del latino, le vocali sono divenute brevi in sillaba implicata (cfr. § 1.3.).

35 E ancora presso / adesso / confesso / fresco (SI 233-6); defendesse / poesse / esse ("E.S. SERE) / devesse (SII 33-6); aspegio / maledegio (MALE + DİCTU) pegio (PĚCTU) / aspegio (SI 873-6); delegio / aspegio / maledegio / alegio (SIII 177-80); questo / asseto (<ASSĒDİTARE) / recreto / creto (SIIl 393-6); creto / breto / recreto / asseto (SI 365-8); cativcto / asscto / foleto / breto (SI 521-4): cfr. Salvioni (1911: 373); Contini (1941:109, $133,130,157,164,113,118)$.

36 Si legge in Salvioni (1975: 6) che “. . . jl dialetto milanese, c già fin dai tempi del Porta, non conosce in realtà che un $o$, l'aperto. Il chiuso c̀ conservato solo nella scrittura, ma c̀, nella

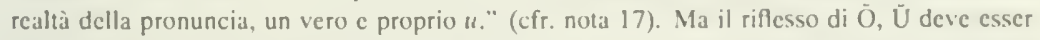
stato in gran parte $[u$ ) già prima del '600. Qucsto scmbra possa dedursi da quanto si legge nel Prissian (Lepschy 1978: 199) che dice a proposito della u (cioc̀ [y]) che " . . à el son strec come l'vltem de s'édu, inn loschan veduro, né mai muda, perché se voùrem dì on son come l'u de amabunt di Latin, e droùuem l'o strec come disenn che fava i Latin antighament che schriueuen amabont par $o \ldots$. . Che la $"$ ". . . mai muda ...." dovrà intendersi nel senso che essa rappresenta il suono $[y]$, c che per $[u]$ si fa ricorso alla grafia $o$, non conic l'intende il Salvioni (1919: 530, n. 1) che essa sia sempre lunga (11 Salvioni sostiene che i termini "largh" - "strec" nel Prissian indichino un opposizione di quantità, non di qualità: cfr. nota 4). Quanto, poi, a amabont, essa rappresenta la forma arcaica di amabumt (con il noto passaggio di $\mathrm{O}$ ad $U$ in sillaba finale implicata: cfr. Nicdermann (1959, 38-42); Pisani 
(1952: 131)).

37 Si noti che questi esempi, come quelli citati sopra a proposito di $e, o$, si riferiscono tutti alla posizione finale. Vien fatto di domandarsi, quindi, se quella norma, citata sopra (§ 2.5.1.), di Isella (" . . la differenza di qualità è anche una differenza di quantità, poiché una vocale aperta è breve e una vocale lunga è chiusa.") abbia valore assoluto e valga sia in parossitonia che in ossitonia. II Salvioni (1975: 5) che pure dà una norma simile (cfr. § 2.4.16.) precisa, però, altrove che "In sillaba aperta, la tonica milanese conosce due lunghezze, quella p. es. di strada e quella di straa e di straat strade; in sillaba chiusa, quella di larga e quella di laark largo, ghi, -ghc. Quella che qui è resa con aa è di più lunga durata." (Salvioni: 1919: 525, n. 1). Se si tien conto, poi, di quell'altra norma, formulata dallo stesso Salvioni (1884: 159) che "... la vocale d'uscita, sparendo dopo consonante sonora rese lunga la vocale accentuata di penultima" (cfr. § 2.4.5.), diventa assai chiaro che relativamente lunga è la tonica (perché appunto tonica!) anche quando è penultima, ma che la lunghezza vocalica vera e propria per il milanese è quclla che si ha in sillaba finale, uscente, o no, in consonante (cfr. $\S \S 2.4 .3$. e 2.3.1.)

38 Pcr han 'hanno,' van 'vanno,' san 'sanno' saran 'saranno,' savaran 'sapranno,' hin 'sono,' ecc. (lsella 1959: 652) con la tonica lunga ma non nasalizzata, cfr. nota 31.

39 Per questa distinzione ci si c̀ basati sulla descrizione che ne dà Beretta (1983: 12): “ . . le /a/ di orientamento anteriore ... e quellc posteriori ...."

40 Corrispondenti, rispettivamente, a peu, jeune in franc., Oefen, öffnen in ted. (Beretta 1983: 17).

41 Si veda, pcrò, Malmberg (1971: 51-2). "Le phénomène qu'on a l'habitude d'appcler 'fester Anschluss' est réscrvé à ccrtaines langues, surtout germaniqucs (spécialcment frappant en allemand), tandis que par exemple les langues romancs ct slaves ne connaitraient que le 'loser Anschluss' (cf. Jespersen, Lehrbuch, p. 204). Et néanmoins, ces langucs connaissent des groupes VOYELLE BREVE + CONSONNE . .. ." Si veda anche Tckavčić (1972: 292-3).

42 Cfr. Battisti (1938: 145): "Rispetto all'apertura laterale avremo forme più forti od cnfatiche in cui si potrà parlare di vera e propria fricativa, e forme più comuni, o leni, in cui il concetto di fricativa è assolutamente superato e in cuj la liquida, con ulteriore lenizione o tende a confondersi con $i$ scmivocalc ... oppure è sostituito da $r$...."

43 Si legge nel Prissian (Lepschy 1978: 198) che la $r$ "No muda mai parpoùst, c s'al'c̀ quai voùlta on tantin più dolza del solit, non la merta saruazion."

44 Per [Y], cfr. nota 16.

45 Si noti che eel 'c̀ lui' c̀ dato con [c:] in Salvioni 1884: 277, con [ع:] in Salvioni 1975: 5.

46 Il Salvioni (1884: 158-61) nota che " . . una sonora (mcdia) non può mai essere consonante d'uscita; è necessario convertasi nella sorda (tenuc) corrispondente. ..." Questa regola, valida in parecchi casi, non c̀ tuttavia senza cccezioni. Lo stesso Salvioni (1884: § 166) trascrive poi maž, pež 'maggio, pcggio' con la fricativa prepalatale sonora; una fricativa sonora si ha, parimcnti, in vōs 'vocc,' nōs 'noce,' crōs 'crocc,' pās 'pacc,' nās 'naso,' rös 'rose' (cfr. § 2.4.7.).

47 Quanto alla posizionc in apparenza contraddittoria del Salvioni, c forse di Isella, al riguardo, cfr. $\S \S 2.4 .19 ., 2.5 .5$.

$48 \mathrm{Va}$ detto qui che Lepschy, in una brcve discussione delle descrizioni del sistema fonetico milanesc, che egli fa scguire al testo del Biffi, intitulato Prissian da Milan della parnonzia milanesa (riprodotto, con alcunc correzioni, nell'cdizione del 1606 in Lepschy 1965, in quella criticamente stabilita da D. Isclla, corredata di una traduzione in italiano con in appendice "Annotazioni al tcsto" dello stesso in Lepschy 1978), da una interpretazione talvolta diversa dei dati (cfr. ad cs., note 17, 20, 31, 46, ccc.), giunge a conclusioni sostanzialmente divergenti dallc mie. Рсr un confronto, si rimanda il lettore a Lepschy 1978: 204-210; 1965: 167-175.

49 Mentre per [e:] [ $\varepsilon$, che si sono generalizzate in sillaba (originariamente) libera e implicata rispettivamentc, si parla di una correlazione fra il timbro chiuso e la quantità lunga da una partc $\mathrm{c}$ il timbro aperto e la quantità breve dall'altra, una siffatta distribuzione e corrispon- 
denza non si ha per $\mid u][0]$. La prima può trovarsi in sillaba sia (originariamente) libera che implicata, per cui non sono pochi i casi in cui $[u \mid$, pur trattandosi di una chiusa, c̀ breve: ross 'rosso," toss 'tosse,' bott 'botte,' ecc.

$50 \mathrm{Cfr}$. nota 20. La mancanza di coppie minime, in questa posizione (in sillaba uscente, cioc̀, in consonante) e forse da attribuirsi al fatto che Ó si riflette in milanese per [s] in sillaba originariamente libera, $\mathrm{e}$ in sillaba implicata per lo più davanti a palatiale: efr. Salvioni 1884: § 43).

51 Sullo stesso argomento, si veda ora Sanga (1984: pp. 60-7); per la quantità vocalica nel bolognese Coco (1970), nel friulano Francescato (1966).

\section{BIBLIOGRAFIA}

Angiolini, F. 1897. Vocabolario milanese-isaliano; rist, anastatica Milano: De Carlo ed., 1978. Ascoli, G.I. 1873. "Saggi ladini." Archivio glottologico italiano, 1:1-537.

Battisti, C. 1938. Fonetica generale. Milano: Hoepli.

Beretta, C. 1980. Contributo per una grammatica del milanese comtemporaneo. Milano: Virgilio ed.

Bertoni, G. 1916. Italia dialettale. Milano: Hocpli; rist. anastatica Milano: Cisalpino Goliardica, 1975.

Cherubini, F. 1839. Vocabolario milanese-italiano. Milano; rist. anastatica Milano: Aldo Martello ed., 1968.

Coco, F. 1970. Il dialetto di Bologna. Bologna: Forni.

Contini, G. 1935. "Saggio d'un'edizione critica di Bonvesin da la Riva." Mem. R. Ist. Lomb., ser. 3, 15: 237-272.

. 1941. Le opere volgari di Bonvesin da la Riva. Roma: Società Filologica Romana.

Durand, M. 1946. Voyelles longues et voyelles brè̀es: éssai sur la nature de la quantité socalique. Paris: Klincksieck.

Francescato, G. 1966. Dialettologia friulana. Udine.

Haudricourt, A. / Juilland, A. 1970 2 . Essai pour une histoire seructurale du phonétisme français. The Hague: Mouton.

Isclla, D. 1959. C. Porta: Poesie. Milano Napoli: Riccardo Ricciardi.

Klausenburger, J. 1975. "Latin Vocalic Quantity to Quality: A Pscudo-Problem?" Diachronic Studies in Romance Linguistics, a cura di M. Saltarelli e D. Wanner. The Hague: Mouton. Lausberg, H. 1947. "Zum romanischen Vokalismus." Romanische Forschungen, 60: 295-307. Lepschy, G.C. 1978. "Una fonologia milanese del 1606: il Prissian da Milan della parnonzia milanesa." Saggi di linguistica iraliana. Bologna: il Mulino; già apparso in L. 'ltalia dialettale, 28 (1965): 143-80.

Lüdtke, H. 1956. Die strukturelle Entwicklung des romanischen Vokalismus. Bonn.

Malmberg, B. 1971. Phonétique générale et romane. The Hague Paris: Mouton.

Muljacic, Z. 1969. Fonologia generale e fonologia della lingua italiana. Bologna: il Mulino.

Nicoli, F. 1983. Grammatica milanese. Busto Arsizio: Bramante ed.

Niedermann, M. $1959_{4}$. Phonétique historique du latin. Paris: Klincksicek.

Pagani, S. 1945. Come parla meneghino. Milano: Ceshina.

Pavia, L. 1928. Nuovi Studî fonico-grafici, filologici, storici, comparativi sulla parlata milanese e suoi connessi. Bergamo.

Pisani, V. 19522. Grammatica latina storica e comparativa. Torino: Rosenberg c Sellier.

Rajna, P. 1881. Il dialetto milanese. Milano: Giuseppe Ottino ed., estratto del volume Milano, a cura di G. Ottino, 1881 .

Rohlfs, G. 1966. Grammatica storica della lingua italiana e dei suoi dialeni: I: Fonetica. Torino: Einaudi.

Salvioni, C. 1911. "Osservazioni sull'antico vocalismo milanese desunte dal metro c dalla rima del cod. Berlinese di Bonvesin da Riva." In Studi letterari e linguistici dedicati a l'in Rajna, 367-388. Firenze. 
. 1919. "Sul dialetto milanese arcaico." Rend. R. Ist. Lomb. ser. 2, 52: 517-40.

1975. "Fonetica e morfologia del dialetto milanese," a cura di D. Isella. L'Italia dialettale, 38: 1-46.

Sanga, G. 1984. Dialettologia lombarda. Pavia.

Sidney-Allen, W. 1965. Vox Latina. Cambridge: Cambridge University Press.

Spence, N.C.W. 1972. "Quantity and Quality in the Vowel System of Vulgar Latin." In Readings in Romance Linguistics, a cura di A. Anderson/Jo Ann Creore, 300-17; già apparso in Word 11 (1965): I-18.

Straka, G. 1959. "Durée et timbre vocaliques: observations de phonétique générale, appliquées à la phonétique historique des langues romanes." Zeitschrift für Phonetik und allgemeine Sprachwissenschaft, 12: 276-300.

Tekavcic, P. 1972. Grammatica storica dell 'italiano. I: Fonematica. Bologna: il Mulino.

Vaananen, V. 1963. Introduction au latin vulgaire. Paris: Klincksieck. . 1965. Évolution et Structure de la langue française. Bern: A. Francke Verlag.

Weinrich, H. $1969_{2}$. Phonologische Studien zur romanischen Sprachgeschichte. Münster Westfallen: Aschendorf. 